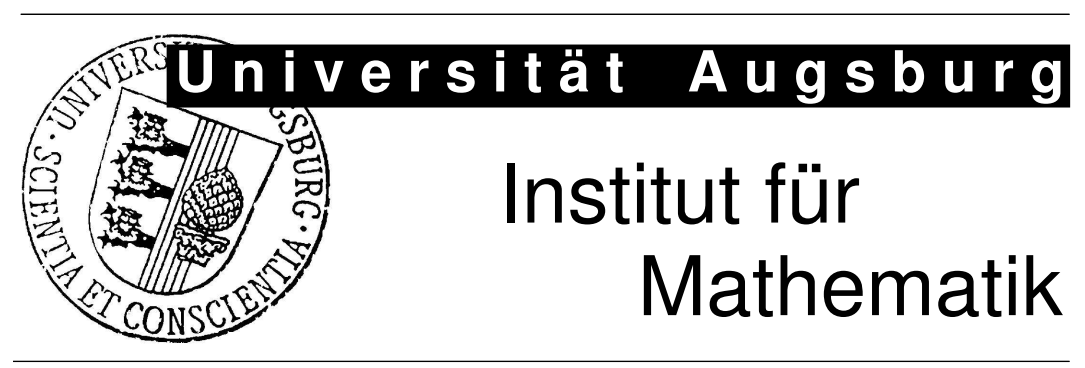

Christoph Kawan, Torben Stender

Growth Rates for Semiflows on Hausdorff Spaces

Preprint Nr. 09/2011 — 06. Juni 2011

Institut für Mathematik, Universitätsstraße, D-86135 Augsburg

http://www.math.uni-augsburg.de/ 


\section{Impressum:}

\section{Herausgeber:}

Institut für Mathematik

Universität Augsburg

86135 Augsburg

http://www.math.uni-augsburg.de/pages/de/forschung/preprints.shtml

\section{ViSdP:}

Christoph Kawan

Institut für Mathematik

Universität Augsburg

86135 Augsburg

Preprint: Sämtliche Rechte verbleiben den Autoren (C) 2011 


\title{
Growth Rates for Semiflows on Hausdorff Spaces
}

\author{
Christoph Kawan · Torben Stender
}

Received: date / Accepted: date

\begin{abstract}
In this paper, we present a theory of vector-valued growth rates for discreteand continuous-time semiflows on Hausdorff spaces. For a given compact flow-invariant set $M$ and an associated growth rate, we introduce the uniform growth spectrum over $M$, and associated real-valued spectra via projections of the vector-valued spectrum onto one-dimensional subspaces. We show that these real-valued spectra are closed intervals if $M$ is additionally connected. We also define the Morse spectrum associated with a growth rate by evaluating the growth rate along chains. Moreover, we relate the uniform growth spectrum to the Morse spectrum and we analyze the meaning of limit sets for the long-time behavior of growth rates.
\end{abstract}

Keywords Semiflows · Growth Rates · Lyapunov Exponents · Morse Spectrum

Mathematics Subject Classification (2000) 37B99 34 D08

\section{Introduction}

In the qualitative theory of dynamical systems, exponential growth rates characterizing the long-time behavior of flows play an important role, in particular, Lyapunov exponents measuring the exponential rate of divergence for nearby trajectories, and rotation numbers measuring the angular rate of rotations (cf., e.g., 1,7,13,14,15, for the latter). In the present paper, we study abstract vector-valued growth rates for semiflows, which generalize both Lyapunov exponents and rotation numbers. Our emphasis is on two spectral concepts for these growth rates and their relations, namely the so-called uniform growth spectrum and the Morse spectrum. Our analysis is essentially based on methods developed in Colonius \& Fabbri \& Johnson [1], Colonius \& Kliemann [2,3] and Lars Grüne [6].

The first author is supported by DFG grant Co 124/17-2 within DFG Priority Program 1305.

Christoph Kawan

Institut für Mathematik, Universität Augsburg, 86159 Augsburg/Germany

E-mail: christoph.kawan@math.uni-augsburg.de

Torben Stender

E-mail: torben.stender@math.uni-augsburg.de 
A growth rate for a semiflow $\Phi: \mathbb{T}_{0}^{+} \times X \rightarrow X\left(\mathbb{T}_{0}^{+} \in\left\{\mathbb{Z}_{0}^{+}, \mathbb{R}_{0}^{+}\right\}\right)$is basically defined as a continuous map $\rho: \mathbb{T}^{+} \times X \rightarrow \mathbb{R}^{m},(t, x) \mapsto \rho^{t}(x)$, such that the map $(t, x) \mapsto t \rho^{t}(x)$ is an additive cocycle over the semiflow $\Phi$, i.e., such that the relation

$$
t_{1} \rho^{t_{1}}(x)+t_{2} \rho^{t_{2}}\left(\Phi\left(t_{1}, x\right)\right)=\left(t_{1}+t_{2}\right) \rho^{t_{1}+t_{2}}(x)
$$

is satisfied for all $t_{1}, t_{2}>0$ and $x \in X$. To develop a spectral theory for such growth rates, we additionally have to impose some boundedness conditions. This fairly general definition, which includes in particular finite-time Lyapunov exponents for linear flows on vector bundles, rotations numbers, and harmonic averages, has already found application in Mezić \& Banaszuk 9] and in Wichtrey [16.

The state space $X$ of the semiflow $\Phi$, in this definition, is only assumed to be a Hausdorff space, not necessarily metrizable. A motivation for working in this general context is that abstract topological spaces arise, e.g., in compactifications of dynamical systems (cf. Patrão [10, and Patrão \& San Martin [11,12]).

For Lyapunov exponents of linear flows on vector bundles, Lars Grüne 6] introduced the uniform exponential spectrum, one of whose motivations lies in the understanding and interpretation of experimental and simulation results. We adapt his definition, and introduce the uniform growth spectrum over a compact invariant set $\mathcal{M}$, for a given growth rate $\rho$. This spectrum is defined as the set of all $\operatorname{limits}_{\lim } \lim _{k \rightarrow \infty} \rho^{t_{k}}\left(x_{k}\right)$ with $x_{k} \in \mathcal{M}$ and $t_{k} \rightarrow \infty$. It is an outer approximation of the Lyapunov spectrum, i.e., the set of $\operatorname{limits}_{\lim } \rightarrow \infty \rho^{t}(x), x \in \mathcal{M}$. In particular, it is a compact set and, in case that $\mathcal{M}$ is connected, each of its projections onto a one-dimensional subspace is a compact interval, whose boundary points are Lyapunov exponents.

We also study the Morse spectrum of a given growth rate over a compact invariant set $\mathcal{M}$, which is, roughly speaking, defined via evaluating the growth rate along chains. This concept was first introduced by Colonius \& Kliemann 2] for linear flows on vector bundles. In the analysis of the Morse spectrum, we mainly adapt methods from Colonius et al. 1 to the more general situation. Additionally assuming connectedness and chain transitivity of $\mathcal{M}$, the Morse spectrum over $\mathcal{M}$ turns out to be a compact and convex set which is the closed convex hull of the Lyapunov spectrum and the convex hull of the uniform exponential spectrum. Moreover, we show that for each point $x$ in a compact invariant set, the limit points $\lim _{k \rightarrow \infty} \rho^{t_{k}}(x)$ are contained in the Morse spectrum over the $\omega$-limit set of $x$.

The present paper is structured as follows: In Section 2 the central notion of growth rates is introduced and several examples are given. In Section 3 , we define the uniform growth spectrum associated with a growth rate and the (real-valued) $z$-related spectra, and we derive some elementary properties of these sets. In the subsequent section 4. we analyze the $z$-related spectra in more detail and prove our first main result, which states that the $z$-related spectrum over a compact connected invariant set is a compact interval, whose boundary points are Lyapunov exponents. Section 5 is devoted to the study of the vector-valued and $z$-related Morse spectra associated with a growth rate. In particular, for connected invariant sets, we prove that the $z$-related Morse spectra coincide with the $z$-related uniform spectra. Moreover, for chain transitive and connected sets we show that the vector-valued Morse spectrum is a compact convex set whose exposed points are Lyapunov exponents. The main result of Section 6 states that all limits of the form $\lim _{k \rightarrow \infty} \rho^{t_{k}}(x)$ are contained in the Morse spectra over $\omega$-limit sets. 
Notation: The notation $\mathbb{T}$ simultaneously stands for the abelian groups $\mathbb{Z}$ (the integers) and $\mathbb{R}$ (the reals). Moreover, we write $\mathbb{T}_{0}^{+}=\{t \in \mathbb{T}: t \geq 0\}, \mathbb{T}_{\geq 1}=\{t \in$ $\mathbb{T}: t \geq 1\}$, and $\mathbb{T}^{+}=\mathbb{T}_{0}^{+} \backslash\{0\}$. If $X$ is a topological space and $A \subset X$, we denote by $\operatorname{cl} A$ the closure of $A$ in $X$. We write $\langle\cdot, \cdot\rangle$ and $\|\cdot\|$ for the Euclidean inner product and its associated norm in $\mathbb{R}^{m}$, respectively. By $B_{r}(x)$ we denote the open ball in $\mathbb{R}^{m}$ centered at $x$ with radius $r$, and by $S^{m-1}$ we denote the unit sphere in $\mathbb{R}^{m}$, $S^{m-1}=\left\{x \in \mathbb{R}^{m}:\|x\|=1\right\}$. The convex hull of a set $A \subset \mathbb{R}^{m}$ is denoted by $\operatorname{co} A$. A semiflow on a topological space $X$ is a continuous mapping $\Phi: \mathbb{T}_{0}^{+} \times X \rightarrow X$ such that $\Phi(0, x)=x$ for all $x \in X$ and $\Phi(t+s, x)=\Phi(s, \Phi(t, x))$ for all $t, s \in \mathbb{T}_{0}^{+}$and $x \in X$. A subset $\mathcal{M} \subset X$ is called invariant for the semiflow $\Phi$ (or $\Phi$-invariant) if $\Phi(t, \mathcal{M}) \subset \mathcal{M}$ holds for all $t \in \mathbb{T}_{0}^{+}$.

\section{Growth Rates}

In this section, we introduce growth rates for semiflows and give some examples. We will frequently consider a semiflow

$$
\Phi: \mathbb{T}_{0}^{+} \times X \rightarrow X
$$

on a Hausdorff space $X$ in this and all subsequent sections.

Definition 1 A growth rate for the semiflow $\Phi$ is a continuous function

$$
\rho: \mathbb{T}^{+} \times X \rightarrow \mathbb{R}^{m}, \quad(t, x) \mapsto \rho^{t}(x),
$$

with the following properties:

1. The function $(t, x) \mapsto t \rho^{t}(x)$ is an additive cocycle over $\Phi$, i.e., for all $t_{1}, t_{2} \in \mathbb{T}^{+}$ and $x \in X$ the relation

$$
t_{1} \rho^{t_{1}}(x)+t_{2} \rho^{t_{2}}\left(\Phi\left(t_{1}, x\right)\right)=\left(t_{1}+t_{2}\right) \rho^{t_{1}+t_{2}}(x)
$$

holds.

2. The restriction of $\rho$ to $\mathbb{T}_{\geq 1} \times X$ is bounded.

3. In case $\mathbb{T}=\mathbb{R}$ : the function

$$
(t, x) \mapsto t \rho^{t}(x), \quad(0,1) \times X \rightarrow \mathbb{R}^{m},
$$

is bounded.

For every vector $z \in S^{m-1}$ we define the mapping

$$
\rho_{z}: \mathbb{T}^{+} \times X \rightarrow \mathbb{R}, \quad(t, x) \mapsto \rho_{z}^{t}(x):=\left\langle\rho^{t}(x), z\right\rangle .
$$

We call $\rho_{z}$ the $z$-related growth rate (associated with $\rho$ ). Moreover, we define

$$
M:=M(\rho):=\max \left\{\sup _{(t, x) \in\left((0,1) \cap \mathbb{T}^{+}\right) \times X}\left\|t \rho^{t}(x)\right\|, \sup _{(t, x) \in \mathbb{T}_{\geq 1} \times X}\left\|\rho^{t}(x)\right\|\right\} .
$$

If for $x \in X$ the limit $\lambda(x):=\lim _{t \rightarrow \infty} \rho^{t}(x)$ exists, we call this limit a Lyapunov exponent (of $\rho$ ). If $\mathcal{M} \subset X$ is a compact $\Phi$-invariant set, the Lyapunov spectrum over $\mathcal{M}$ is defined as the set of all Lyapunov exponents

$$
\Sigma_{\mathrm{Ly}, \Phi, \rho}(\mathcal{M}):=\{\lambda(x): x \in \mathcal{M}, \lambda(x) \text { exists }\} .
$$

If it is clear from the context what $\Phi$ and $\rho$ are, we omit the corresponding indices. 
The following lemma shows a relation between the long-time behavior of a semiflow and that of its associated growth rates.

Lemma 1 Let $\rho: \mathbb{T}^{+} \times X \rightarrow \mathbb{R}^{m}$ be a growth rate for the semiflow $\Phi$ and let $t_{k}$ be a sequence in $\mathbb{T}^{+}$with $t_{k} \rightarrow \infty$. If $\lambda=\lim _{k \rightarrow \infty} \rho^{t_{k}}(x)$ exists for some $x \in X$, then for any $t \in \mathbb{T}^{+}$it holds that $\lambda=\lim _{k \rightarrow \infty} \rho^{t_{k}-t}(\Phi(t, x))$, where the sequence is only defined for $k$ large enough such that $t_{k}-t>0$. The same holds for $z$-related growth rates.

Proof For $t_{1}=t$ and $t_{2}=t_{k}-t$, the definition of growth rates implies

$$
\rho^{t_{k}-t}(\Phi(t, x))=\frac{1}{t_{k}-t}\left[t_{k} \rho^{t_{k}}(x)-t \rho^{t}(x)\right]=\frac{1}{1-\frac{t}{t_{k}}} \rho^{t_{k}}(x)-\frac{t}{t_{k}-t} \rho^{t}(x) .
$$

From this identity the assertion immediately follows. For $z$-related growth rates the proof works analogously.

Example 1 The following example shows that the (classical) finite-time Lyapunov exponents of a linear flow on a vector bundle define a real-valued growth rate for the induced flow on the associated projective bundle: Consider a linear flow $\psi: \mathbb{R} \times \mathcal{V} \rightarrow \mathcal{V}$ on a vector bundle $\pi: \mathcal{V} \rightarrow B$ with compact metric base space $B$ and base flow $\Theta: \mathbb{R} \times B \rightarrow B$. Assume that we are given a norm on $\mathcal{V}$, i.e., a family of norms $\|\cdot\|_{b}$ on the fibers $\mathcal{V}_{b}$, varying continuously with $b \in B$. Let $\mathbb{P} \pi: \mathbb{P} \mathcal{V} \rightarrow B$ be the associated projective bundle with the induced flow $\Phi=\mathbb{P} \psi: \mathbb{R} \times \mathbb{P V} \rightarrow \mathbb{P} \mathcal{V}$, and define

$$
\rho^{t}(\mathbb{P} v):=\frac{1}{t} \log \left\|\psi\left(t, \frac{v}{\|v\|}\right)\right\|, \quad \rho: \mathbb{R}^{+} \times \mathbb{P} \mathcal{V} \rightarrow \mathbb{R} .
$$

This map is easily seen to be well-defined and continuous. Moreover, we have

$$
\begin{aligned}
t_{1} \rho^{t_{1}}(\mathbb{P} v) & +t_{2} \rho^{t_{2}}\left(\Phi\left(t_{1}, \mathbb{P} v\right)\right) \\
& =\log \left\|\psi\left(t_{1}, \frac{v}{\|v\|}\right)\right\|+\log \left\|\psi\left(t_{2}, \frac{\psi\left(t_{1}, v\right)}{\left\|\psi\left(t_{1}, v\right)\right\|}\right)\right\| \\
& =\log \left\|\psi\left(t_{1}, v\right)\right\|-\log \|v\|+\log \left\|\psi\left(t_{2}, \psi\left(t_{1}, v\right)\right)\right\|-\log \left\|\psi\left(t_{1}, v\right)\right\| \\
& =\log \left\|\psi\left(t_{1}+t_{2}, \frac{v}{\|v\|}\right)\right\|=\left(t_{1}+t_{2}\right) \rho^{t_{1}+t_{2}}(\mathbb{P} v) .
\end{aligned}
$$

The function $\rho$ is bounded on $\mathbb{R}_{>1} \times \mathbb{P} \mathcal{V}$, since there are constants $K \geq 1$ and $\alpha>0$ such that (cf. Colonius et al. [1, Rem. 2.1])

$$
\frac{1}{K} e^{-\alpha t} \leq\left\|\psi\left(t, \frac{v}{\|v\|}\right)\right\| \leq K e^{\alpha t}
$$

for all $t \geq 0$ and $v \in \mathcal{V},\|v\| \neq 0$, which implies

$$
-\log (K)-\alpha \leq \rho^{t}(\mathbb{P} v) \leq \log (K)+\alpha \text { for all } t \geq 1, \mathbb{P} v \in \mathbb{P} \mathcal{V} .
$$

Finally, for $t \in(0,1)$ we have

$$
-\log (K)-\alpha \leq t \rho^{t}(\mathbb{P} v) \leq \log (K)+\alpha .
$$

Hence, $\rho$ is a growth rate for $\Phi$ with $M(\rho) \leq \log (K)+\alpha$. 
Example 2 Consider a bilinear control system

$$
\dot{x}(t)=\left[A_{0}+\sum_{i=1}^{m} u_{i}(t) A_{i}\right] x(t)=A(u(t)) x(t), \quad u \in \mathcal{U},
$$

on $\mathbb{R}^{d}$ with admissible control functions

$$
\mathcal{U}=\left\{u: \mathbb{R} \rightarrow \mathbb{R}^{m}: u \text { measurable with } u(t) \in U \text { a.e. }\right\},
$$

where $U \subset \mathbb{R}^{m}$ is a compact and convex set. The unique solution with initial value $x$ for the control function $u$ is denoted by $\varphi(t, x, u)$. These solutions define a continuous skew-product flow on $\mathcal{U} \times \mathbb{R}^{d}$, the so-called control flow, by

$$
\psi: \mathbb{R} \times\left(\mathcal{U} \times \mathbb{R}^{d}\right) \rightarrow \mathcal{U} \times \mathbb{R}^{d}, \quad(t,(u, x)) \mapsto(u(t+\cdot), \varphi(t, x, u)),
$$

where $\mathcal{U}$ is endowed with the weak ${ }^{*}$-topology of $L^{\infty}\left(\mathbb{R}, \mathbb{R}^{m}\right)=L^{1}\left(\mathbb{R}, \mathbb{R}^{m}\right)^{*}$, which makes it a compact metrizable space. Here, we are interested in the induced flow on $\mathcal{U} \times \mathrm{St}_{2} \mathbb{R}^{d}$, where $\mathrm{St}_{2} \mathbb{R}^{d}$ is the Stiefel manifold of orthonormal 2-frames in $\mathbb{R}^{d}$. This flow is given by

$$
\begin{aligned}
\Phi: \mathbb{R} \times\left(\mathcal{U} \times \mathrm{St}_{2} \mathbb{R}^{d}\right) & \rightarrow \mathcal{U} \times \mathrm{St}_{2} \mathbb{R}^{d}, \\
\left(t,\left(u,\left(x_{0}, y_{0}\right)\right)\right) & \mapsto\left(u(t+\cdot), x_{t}\left(x_{0}, u\right), y_{t}\left(x_{0}, y_{0}, u\right)\right),
\end{aligned}
$$

where $x_{t}\left(x_{0}, u\right), y_{t}\left(x_{0}, y_{0}, u\right)$ are defined via Gram-Schmidt orthonormalization of $\left(\varphi\left(t, x_{0}, u\right), \varphi\left(t, y_{0}, u\right)\right)$. For this flow, a growth rate rot : $\mathbb{R}^{+} \times\left(\mathcal{U} \times \mathrm{St}_{2} \mathbb{R}^{d}\right) \rightarrow \mathbb{R}$ can be defined, which measures the rotational behaviour of the solutions $\varphi(t, x, u)$. This growth rate is given by

$$
\operatorname{rot}^{t}\left(u,\left(x_{0}, y_{0}\right)\right):=\frac{1}{t} \int_{0}^{t}\left\langle A(u(t)) x_{t}\left(x_{0}, u\right), y_{t}\left(x_{0}, y_{0}, u\right)\right\rangle d t .
$$

The numbers $\operatorname{rot}^{t}\left(u,\left(x_{0}, y_{0}\right)\right)$ are called (finite-time) rotation numbers. Relation (1) is verified using the flow property of $\Phi$ :

$$
\begin{aligned}
\left(t_{1}+t_{2}\right) & \operatorname{rot}^{t_{1}+t_{2}}\left(u,\left(x_{0}, y_{0}\right)\right)=\int_{0}^{t_{1}+t_{2}}\left\langle A(u(t)) x_{t}\left(x_{0}, u\right), y_{t}\left(x_{0}, y_{0}, u\right)\right\rangle d t \\
= & \int_{0}^{t_{1}}\left\langle A(u(t)) x_{t}\left(x_{0}, u\right), y_{t}\left(x_{0}, y_{0}, u\right)\right\rangle d t \\
& +\int_{0}^{t_{2}}\left\langle A\left(u\left(t+t_{1}\right)\right) x_{t+t_{1}}\left(x_{0}, u\right), y_{t+t_{1}}\left(x_{0}, y_{0}, u\right)\right\rangle d t \\
& =t_{1} \operatorname{rot}^{t_{1}}\left(u,\left(x_{0}, y_{0}\right)\right) \\
& +\int_{0}^{t_{2}}\left\langle A\left(u\left(t+t_{1}\right)\right) x_{t}\left(x_{t}\left(x_{0}, u\right), u\left(t_{1}+\cdot\right)\right), y_{t}\left(x_{t}\left(x_{0}, u\right), y_{t}\left(y_{0}, u\right), u\left(t_{1}+\cdot\right)\right)\right\rangle d t \\
& =t_{1} \rho^{t_{1}}\left(u,\left(x_{0}, y_{0}\right)\right)+t_{2} \rho^{t_{2}}\left(\Phi\left(t_{1},\left(u,\left(x_{0}, y_{0}\right)\right)\right)\right) .
\end{aligned}
$$

Standard estimates lead to

$$
\left|\operatorname{rot}^{t}\left(u,\left(x_{0}, y_{0}\right)\right)\right| \leq \max _{u \in U}\|A(u)\| \text { for all } t>0 .
$$

For continuity of rot and a detailed analysis of rotation numbers we refer to Stender [15. 
Example 3 In this example, we describe the growth rates which are studied in Colonius et al. 1. Let $\psi: \mathbb{R} \times X \rightarrow X$ be a continuous flow on a fiber bundle $\pi: X \rightarrow B$ with compact base space $B$, and let $f: X \rightarrow \mathbb{R}^{m}$ be a continuous function. Assume that the map

$$
f_{B}: \mathbb{R} \times B \rightarrow \mathbb{R}^{m}, \quad(t, b) \mapsto f(\psi(t, x))-f(x), \quad x \in \pi^{-1}(b),
$$

is well-defined, i.e., that $f_{B}(t, b)$ is independent of the choice of $x \in \pi^{-1}(b)$. Define

$$
\rho: \mathbb{R}^{+} \times B \rightarrow \mathbb{R}^{m}, \quad(t, b) \mapsto \frac{1}{t} f_{B}(t, b) .
$$

Then $\rho$ is a growth rate for the induced flow $\Phi$ on the base space $B$. Continuity of $\rho$ follows from the facts that $(t, x) \mapsto f(\psi(t, x))-f(x)$ is continuous and $\pi$ is a projection map (cf. 1, Lem. 2.3]). The relation (1) follows easily:

$$
\begin{aligned}
\left(t_{1}+t_{2}\right) \rho^{t_{1}+t_{2}}(b) & =f_{B}\left(t_{1}+t_{2}, b\right)=f\left(\psi\left(t_{2}, \psi\left(t_{1}, x\right)\right)\right)-f(x) \\
& =f\left(\psi\left(t_{2}, \psi\left(t_{1}, x\right)\right)\right)-f\left(\psi\left(t_{1}, x\right)\right)+f\left(\psi\left(t_{1}, x\right)\right)-f(x) \\
& =f_{B}\left(t_{2}, \Phi\left(t_{1}, b\right)\right)+f_{B}\left(t_{1}, b\right) \\
& =t_{2} \rho^{t_{2}}\left(\Phi\left(t_{1}, b\right)\right)+t_{1} \rho^{t_{1}}(b) .
\end{aligned}
$$

By compactness of $B$ and continuity of $f_{B}$, also the required boundedness assumptions for growth rates follow (cf. Example 4). This general construction in particular includes Lyapunov exponents as described in Example 1 Here $X$ is the complement of the zero section in the total space of a vector bundle (with compact base space), $B$ is the total space of the associated projective bundle, and $\pi: X \rightarrow B$ is the natural projection. The function $f$ is given by $f(x)=\log \|x\|$.

Example 4 In San Martin \& Seco 8, vector-valued Lyapunov and Morse exponents for flows on principal bundles are analyzed. The general construction is as follows: Let $E$ be a compact Hausdorff space and $\Phi: \mathbb{T} \times E \rightarrow E$ a continuous flow $(\mathbb{T} \in\{\mathbb{Z}, \mathbb{R}\})$. If $V$ is a finite-dimensional normed vector space, a $V$-valued cocycle over $E$ is a continuous map $a: \mathbb{T} \times E \rightarrow V$ with

$$
a(t+s, x)=a(t, \Phi(s, x))+a(s, x) \text { for all } t, s \in \mathbb{T}, x \in E .
$$

Given $x \in E$ and $t \in \mathbb{T}^{+}$, the finite-time Lyapunov exponent of the cocycle $a$ at $(x, t)$ is

$$
\lambda^{t}(x):=\frac{1}{t} a(t, x)
$$

Taking $V=\mathbb{R}^{m}$, this defines a growth rate in the sense of our definition. Continuity is clear and the relation (1) immediately follows from the cocycle property (3). Moreover, from (3) we conclude (for $\mathbb{T}=\mathbb{R}$, without loss of generality), writing $t=n+r \geq 1$ with $n \in \mathbb{Z}^{+}$and $r \in[0,1)$,

$$
\begin{aligned}
\left\|\lambda^{t}(x)\right\| & =\frac{1}{n+r}\|a(n+r, x)\| \leq \frac{1}{n+r}\left(\|a(r, \Phi(n, x))\|+\sum_{k=0}^{n-1}\|a(1, \Phi(k, x))\|\right) \\
& \leq \frac{n+1}{n+r} \max _{(s, x) \in[0,1] \times X}\|a(s, x)\| \leq 2 \max _{(s, x) \in[0,1] \times X}\|a(s, x)\|<\infty,
\end{aligned}
$$

and

$$
\left\|t \lambda^{t}(x)\right\|=\|a(t, x)\| \leq \max _{(s, x) \in[0,1] \times X}\|a(s, x)\| \text { for all }(t, x) \in(0,1] \times X .
$$


In [8], continuous flows on principal bundles with semisimple structure group $G$ are investigated. Using the Iwasawa decomposition $G=K A N$, an additive cocycle over the flow taking values in $\mathfrak{a}=\log A$ can be constructed and its Lyapunov and Morse exponents are studied.

Example 5 Let $X$ be a metric space and $\psi: \mathbb{R}_{0}^{+} \times X \rightarrow X$ a semiflow on $X$. For some $\omega \in \mathbb{R}$, consider the semiflow

$$
\Phi^{\omega}: \mathbb{R}_{0}^{+} \times\left(S^{1} \times X\right) \rightarrow S^{1} \times X, \quad(t,(z, x)) \mapsto\left(e^{i \omega t} z, \psi(t, x)\right) .
$$

Here, the unit circle $S^{1}$ is regarded as a subset of the complex plane $\mathbb{C}$. Let $f: X \rightarrow \mathbb{C}$ be a bounded continuous function and define

$$
\rho^{t}(z, x):=z \frac{1}{t} \int_{0}^{t} e^{i \omega t} f\left(\psi_{t}(x)\right) d t
$$

It can easily be verified that $\rho$ satisfies (1) for the semiflow $\Phi^{\omega}$. Growth rates of this form can be used to study the rotational behavior of dynamical systems. They, together with their spectral sets, are studied in Wichtrey [16], not only in the continuous but also in a measurable setting.

\section{The Uniform Growth Spectrum}

In the setting of the preceding section, we now introduce the uniform growth spectrum over a compact $\Phi$-invariant set for a given growth rate. This definition generalizes Grüne's definition of the uniform exponential spectrum for linear flows on vector bundles (cf. [6, Def. 3.1]).

Definition 2 Let $\mathcal{M} \subset X$ be a compact $\Phi$-invariant set and $\rho: \mathbb{T}^{+} \times X \rightarrow \mathbb{R}^{m}$ a growth rate for $\Phi$. The uniform growth spectrum over $\mathcal{M}$ (associated with $\rho$ ) is defined by

$$
\Sigma_{\mathrm{UG}, \Phi, \rho}(\mathcal{M}):=\left\{\lambda \in \mathbb{R}^{m}: \begin{array}{c}
\exists t_{k} \rightarrow \infty \text { and } x_{k} \in \mathcal{M} \\
\text { such that } \lim _{k \rightarrow \infty} \rho^{t_{k}}\left(x_{k}\right)=\lambda
\end{array}\right\} .
$$

For $z \in S^{m-1}$, the $z$-related uniform growth spectrum over $\mathcal{M}$ is defined by

$$
\Sigma_{\mathrm{UG}, \Phi, \rho}^{z}(\mathcal{M}):=\left\{\lambda \in \mathbb{R}: \begin{array}{c}
\exists t_{k} \rightarrow \infty \text { and } x_{k} \in \mathcal{M} \\
\text { such that } \lim _{k \rightarrow \infty} \rho_{z}^{t_{k}}\left(x_{k}\right)=\lambda
\end{array}\right\} .
$$

If it is clear from the context what $\Phi$ and $\rho$ are, we omit the corresponding indices.

By boundedness of $\rho$ on $\mathbb{T}_{\geq 1} \times X$, it is clear that $\Sigma_{\mathrm{UG}}(\mathcal{M})$ and $\Sigma_{\mathrm{UG}}^{z}(\mathcal{M})$ are nonempty if $\mathcal{M}$ is nonempty. The following lemma can be proved by easy topological arguments, hence we omit the proof.

Lemma 2 It holds that

$$
\Sigma_{\mathrm{UG}}(\mathcal{M})=\bigcap_{T \in \mathbb{T}^{+}} \mathrm{cl} \bigcup_{t \geq T} \rho^{t}(\mathcal{M}) \text { and } \Sigma_{\mathrm{UG}}^{z}(\mathcal{M})=\bigcap_{T \in \mathbb{T}^{+}} \mathrm{cl} \bigcup_{t \geq T} \rho_{z}^{t}(\mathcal{M}) .
$$

Corollary 1 If $\mathbb{T}=\mathbb{R}$ and $\mathcal{M}$ is connected, then also $\Sigma_{\mathrm{UG}}(\mathcal{M})$ and $\Sigma_{\mathrm{UG}}^{z}(\mathcal{M})$ are connected. 
Proof This follows by the same arguments which are used to prove that $\omega$-limit sets of continuous-time systems on compact spaces are connected.

As the next proposition shows, in the continuous-time case it is sufficient to consider time sequences $t_{k} \rightarrow \infty$ with $t_{k} \in \mathbb{Z}^{+}$in the definition of $\Sigma_{\mathrm{UG}}(\mathcal{M})$.

Proposition 1 Assume that $\mathbb{T}=\mathbb{R}$ in Definition 2, Then

$$
\Sigma_{\mathrm{UG}}(\mathcal{M})=\left\{\lambda \in \mathbb{R}^{m}: \begin{array}{c}
\exists t_{k} \in \mathbb{Z}^{+}, t_{k} \rightarrow \infty, \text { and } x_{k} \in \mathcal{M} \\
\text { such that } \lim _{k \rightarrow \infty} \rho^{t_{k}}\left(x_{k}\right)=\lambda
\end{array}\right\}
$$

The analogous statement holds for the z-related uniform growth spectrum.

Proof Consider sequences $t_{k} \in \mathbb{R}^{+}, t_{k} \rightarrow \infty$, and $x_{k} \in \mathcal{M}$ such that the limit $\lambda=$ $\lim _{k \rightarrow \infty} \rho^{t_{k}}\left(x_{k}\right)$ exists. We write each $t_{k}$ as $t_{k}=\left\lfloor t_{k}\right\rfloor+\tau_{k}$, where $\left\lfloor t_{k}\right\rfloor$ denotes the integer part of $t_{k}$ and $\tau_{k}=t_{k}-\left\lfloor t_{k}\right\rfloor \in[0,1)$. Since $t_{k} \rightarrow \infty$, we may assume that $\left\lfloor t_{k}\right\rfloor \geq 1$ for all $k$. For all $k$ with $t_{k} \notin \mathbb{Z}^{+}$, i.e., $\tau_{k}>0$, the definition of growth rates implies

$$
\tau_{k} \rho^{\tau_{k}}\left(x_{k}\right)+\left\lfloor t_{k}\right\rfloor \rho^{\left\lfloor t_{k}\right\rfloor}\left(\Phi\left(\tau_{k}, x_{k}\right)\right)=t_{k} \rho^{t_{k}}\left(x_{k}\right) .
$$

Defining a sequence $y_{k} \in \mathcal{M}$ by $y_{k}:=\Phi\left(\tau_{k}, x_{k}\right)$ for all $k$, we obtain

$$
\rho^{\left\lfloor t_{k}\right\rfloor}\left(y_{k}\right)=\left\{\begin{array}{cl}
\rho^{t_{k}}\left(x_{k}\right) & \text { if } \tau_{k}=0, \\
\frac{t_{k}}{\left\lfloor t_{k}\right\rfloor} \rho^{t_{k}}\left(x_{k}\right)-\frac{1}{\left\lfloor t_{k}\right\rfloor} \tau_{k} \rho^{\tau_{k}}\left(x_{k}\right) & \text { if } \tau_{k}>0 .
\end{array}\right.
$$

Since $t_{k} /\left\lfloor t_{k}\right\rfloor \rightarrow 1,1 /\left\lfloor t_{k}\right\rfloor \rightarrow 0$, and $\left|\tau_{k} \rho^{\tau_{k}}\left(x_{k}\right)\right| \leq M$, we have $\lim _{k \rightarrow \infty} \rho^{\left\lfloor t_{k}\right\rfloor}\left(y_{k}\right)=\lambda$, which implies the assertion. For the $z$-related spectrum the proof works analogously.

The above proposition justifies to consider only the discrete-time case in the analysis of the uniform growth spectrum. Hence, in the rest of this section we assume that $\mathbb{T}=\mathbb{Z}$. The next two lemmas are modifications of Grüne [6. Lem. 2.3 and Lem. 2.4].

Lemma 3 Let $\rho: \mathbb{Z}^{+} \times X \rightarrow \mathbb{R}^{m}$ be a growth rate for the semiflow $\Phi$. Then for all $t_{1}, t_{2} \in \mathbb{Z}^{+}$and $x \in X$ we have

$$
\left\|\rho^{t_{1}+t_{2}}(x)-\rho^{t_{1}}(x)\right\| \leq 2 M \frac{t_{2}}{t_{1}+t_{2}},
$$

with $M$ defined as in (2). The same inequality holds for $z$-related growth rates.

Proof It follows directly from the definition of growth rates that

$$
\begin{aligned}
\left\|\rho^{t_{1}+t_{2}}(x)-\rho^{t_{1}}(x)\right\| & =\left\|\frac{1}{t_{1}+t_{2}}\left(t_{2} \rho^{t_{2}}\left(\Phi\left(t_{1}, x\right)\right)+t_{1} \rho^{t_{1}}(x)\right)-\rho^{t_{1}}(x)\right\| \\
& \leq \frac{t_{2}}{t_{1}+t_{2}}\left\|\rho^{t_{2}}\left(\Phi\left(t_{1}, x\right)\right)\right\|+\left|\frac{t_{1}}{t_{1}+t_{2}}-1\right|\left\|\rho^{t_{1}}(x)\right\| \\
& =\left(\left\|\rho^{t_{2}}\left(\Phi\left(t_{1}, x\right)\right)\right\|+\left\|\rho^{t_{1}}(x)\right\|\right) \frac{t_{2}}{t_{1}+t_{2}} \leq 2 M \frac{t_{2}}{t_{1}+t_{2}} .
\end{aligned}
$$

For $z$-related growth rates the corresponding inequality follows with Cauchy-Schwarz. 
Lemma 4 Let $\rho: \mathbb{Z}^{+} \times X \rightarrow \mathbb{R}^{m}$ be a nonzero growth rate for the semiflow $\Phi$. Fix $z \in S^{m-1}$ and let $x \in X, t \in \mathbb{Z}^{+}$. Then for any $\varepsilon \in(0,2 M)$ there is a time $t_{1} \leq[(2 M-\varepsilon) t] /(2 M)$ such that

$$
\rho_{z}^{s}\left(\Phi\left(t_{1}, x\right)\right) \leq \rho_{z}^{t}(x)+\varepsilon \text { for all } s \in\left(0, t-t_{1}\right] \cap \mathbb{Z},
$$

where $M$ is defined as in (2). Furthermore, $t-t_{1} \geq \varepsilon t /(2 M) \rightarrow \infty$ for $t \rightarrow \infty$.

Proof For brevity, we write $\sigma:=\rho_{z}^{t}(x)$. Let $\varepsilon \in(0,2 M)$ and define

$$
\beta:=\max _{s \in(0, t] \cap \mathbb{Z}} \rho_{z}^{s}(x) .
$$

If $\beta \leq \sigma+\varepsilon$, the assertion follows with $t_{1}=0$. For $\beta>\sigma+\varepsilon$ let

$$
t_{1}:=\max \left\{s \in(0, t] \cap \mathbb{Z}: \rho_{z}^{s}(x) \geq \sigma+\varepsilon\right\} .
$$

Then we have

$$
\rho_{z}^{t_{1}}(x) \geq \sigma+\varepsilon
$$

We use Lemma 3 with $t_{1}$ and $t_{2}=t-t_{1}$ to obtain

$$
\varepsilon \leq \rho_{z}^{t_{1}}(x)-\sigma=\rho_{z}^{t_{1}}(x)-\rho_{z}^{t}(x)=\left|\rho_{z}^{t_{1}}(x)-\rho_{z}^{t}(x)\right| \leq 2 M \frac{t-t_{1}}{t} .
$$

This implies $t-t_{1} \geq \varepsilon t /(2 M)$. Now, let $s \in\left(0, t-t_{1}\right] \cap \mathbb{Z}$. Then $s+t_{1} \in\left(t_{1}, t\right]$, and hence $\rho_{z}^{s+t_{1}}(x)<\sigma+\varepsilon$. Using the definition of growth rates, this implies

$$
\begin{aligned}
\rho_{z}^{s}\left(\Phi\left(t_{1}, x\right)\right) & =\frac{1}{s}\left[\left(t_{1}+s\right) \rho_{z}^{t_{1}+s}(x)-t_{1} \rho_{z}^{t_{1}}(x)\right] \\
& <\frac{1}{s}\left[\left(t_{1}+s\right)(\sigma+\varepsilon)-t_{1}(\sigma+\varepsilon)\right] \\
& =\sigma+\varepsilon=\rho_{z}^{t}(x)+\varepsilon,
\end{aligned}
$$

which finishes the proof.

Proposition 2 Let $\mathcal{M} \subset X$ be a compact $\Phi$-invariant set, $\rho: \mathbb{Z}^{+} \times X \rightarrow \mathbb{R}^{m}$ a growth rate for $\Phi$, and $z \in S^{m-1}$. Then

$$
\left\langle\Sigma_{\mathrm{UG}}(\mathcal{M}), z\right\rangle=\Sigma_{\mathrm{UG}}^{z}(\mathcal{M}) .
$$

Proof To show the inclusion " $\subset$ ", let $\lambda \in\left\langle\Sigma_{\mathrm{UG}}(\mathcal{M}), z\right\rangle$. Then there are sequences $t_{k} \rightarrow \infty$ and $x_{k} \in \mathcal{M}$ such that $\lambda=\langle\mu, z\rangle$ with $\mu:=\lim _{k \rightarrow \infty} \rho^{t_{k}}\left(x_{k}\right)$. Hence,

$$
\lambda=\langle\mu, z\rangle=\left\langle\lim _{k \rightarrow \infty} \rho_{z}^{t_{k}}\left(x_{k}\right), z\right\rangle=\lim _{k \rightarrow \infty}\left\langle\rho_{z}^{t_{k}}\left(x_{k}\right), z\right\rangle .
$$

For the converse inclusion, let $\lambda \in \Sigma_{\mathrm{UG}}^{z}(\mathcal{M})$. Then there are sequences $t_{k} \rightarrow \infty$ and $x_{k} \in \mathcal{M}$ such that $\lambda=\lim _{k \rightarrow \infty}\left\langle\rho^{t_{k}}\left(x_{k}\right), z\right\rangle$. Since $\rho$ is bounded, there is a convergent subsequence $\rho^{t_{k_{l}}}\left(x_{k_{l}}\right) \rightarrow \mu \in \Sigma_{\mathrm{UG}}(\mathcal{M})$, which implies $\lambda=\langle\mu, z\rangle \in\left\langle\Sigma_{\mathrm{UG}}(\mathcal{M}), z\right\rangle$.

As the next proposition shows, the uniform growth spectrum and the $z$-related growth spectra are compact sets.

Proposition 3 Let $\mathcal{M} \subset X$ be a compact $\Phi$-invariant set and $\rho: \mathbb{Z}^{+} \times X \rightarrow \mathbb{R}^{m}$ a growth rate for $\Phi$. Then $\Sigma_{\mathrm{UG}}(\mathcal{M})$ and $\Sigma_{\mathrm{UG}}^{z}(\mathcal{M})$ (for any $z \in S^{m-1}$ ) are compact sets. 
Proof Since $\rho$ is bounded, also $\Sigma_{\mathrm{UG}}(\mathcal{M})$ is bounded. Now, consider a sequence $\lambda_{k}$ in $\Sigma_{\mathrm{UG}}(\mathcal{M})$ such that the limit $\lambda:=\lim _{k \rightarrow \infty} \lambda_{k}$ exists. For each $k$ there are $x_{k} \in \mathcal{M}$ and $t_{k} \geq 1$ with $\left\|\rho^{t_{k}}\left(x_{k}\right)-\lambda_{k}\right\| \leq 2^{-k}$ implying

$$
\left\|\lambda-\rho^{t_{k}}\left(x_{k}\right)\right\| \leq\left\|\lambda-\lambda_{k}\right\|+\left\|\lambda_{k}-\rho^{t_{k}}\left(x_{k}\right)\right\| \leq\left\|\lambda-\lambda_{k}\right\|+\frac{1}{2^{k}} .
$$

Hence, $\rho^{t_{k}}\left(x_{k}\right)$ converges to $\lambda$, which proves that $\Sigma_{\mathrm{UG}}(\mathcal{M})$ is closed. For $\Sigma_{\mathrm{UG}}^{z}(\mathcal{M})$ the proof works analogously.

\section{Properties of the Real-Valued Spectra}

In this section, we analyze the $z$-related spectra over a connected compact $\Phi$-invariant set. Again, without loss of generality, we only consider the discrete-time case. The following theorem is a straightforward generalization of Grüne 6, Thm. 3.3]. It shows that the $z$-related spectra are compact intervals whose boundary points are Lyapunov exponents.

Theorem 1 Let $\mathcal{M} \subset X$ be a connected compact $\Phi$-invariant set, $\rho: \mathbb{Z}^{+} \times X \rightarrow \mathbb{R}^{m}$ a growth rate for $\Phi$, and $z \in S^{m-1}$. Then there are real numbers $\rho_{*} \leq \rho^{*}$ such that

$$
\Sigma_{\mathrm{UG}}^{z}(\mathcal{M})=\left[\rho_{*}, \rho^{*}\right] .
$$

Moreover, there are $x_{*}, x^{*} \in \mathcal{M}$ such that $\rho_{z}^{t}\left(x_{*}\right) \leq \rho_{*}$ and $\rho_{z}^{t}\left(x^{*}\right) \geq \rho^{*}$ for all $t \geq 1$ and

$$
\lim _{t \rightarrow \infty} \rho_{z}^{t}\left(x_{*}\right)=\rho_{*}, \quad \lim _{t \rightarrow \infty} \rho_{z}^{t}\left(x^{*}\right)=\rho^{*} .
$$

Proof Compactness of $\Sigma_{\mathrm{UG}}^{z}(\mathcal{M})$ is guaranteed by Proposition 3 . If $\rho \equiv 0$, the proof is trivial. Hence, we may assume that $\rho$ is not identically zero. The rest of the proof is subdivided into two steps.

Step 1. Let $\rho_{*}:=\min \Sigma_{\mathrm{UG}}^{z}(\mathcal{M})$. We first show the existence of $x_{*}$. By definition of $\Sigma_{\mathrm{UG}}^{z}(\mathcal{M})$, there are sequences $x_{k} \in \mathcal{M}$ and $t_{k} \rightarrow \infty$ with

$$
\rho_{z}^{t_{k}}\left(x_{k}\right)<\rho_{*}+\varepsilon_{k},
$$

where $\varepsilon_{k} \searrow 0$. Let $\widetilde{\varepsilon}_{k}:=1 / \sqrt{t_{k}}$. By applying Lemma 4 to $x_{k}$ and $t_{k}$ with $\varepsilon=\widetilde{\varepsilon}_{k}$ for each $k$ large enough such that $\widetilde{\varepsilon}_{k}<2 M$, we obtain times $t_{k}^{*}$ such that

$$
\rho_{z}^{s}\left(\Phi\left(t_{k}^{*}, x_{k}\right)\right) \leq \rho_{*}+\varepsilon_{k}+\widetilde{\varepsilon}_{k} \text { for all } s \in\left(0, t_{k}-t_{k}^{*}\right] \cap \mathbb{Z},
$$

where $t_{k}-t_{k}^{*} \geq \widetilde{\varepsilon}_{k} t_{k} /(2 M)=\sqrt{t_{k}} /(2 M)$. We let $\widetilde{x}_{k}:=\Phi\left(t_{k}^{*}, x_{k}\right)$ and $\widetilde{t}_{k}:=t_{k}-t_{k}^{*} \rightarrow \infty$. Since $\mathcal{M}$ is compact, there is a convergent subnet of the sequence $\left(\widetilde{x}_{k}\right)_{k \in \mathbb{Z}^{+}}$(considered as a net), converging to some $\widetilde{x} \in \mathcal{M}$ (cf. Willard [17, Thm. 11.5 and Thm. 17.4]). That is, there is a directed set $(E, \geq)$ and a function $f: E \rightarrow \mathbb{Z}^{+}$with $e_{1} \leq e_{2}$ implying $f\left(e_{1}\right) \leq f\left(e_{2}\right)$ such that for every $n \in \mathbb{Z}^{+}$there is $e \in E$ with $f(e) \geq n$ and such that for every neighborhood $U$ of $\widetilde{x}$ there is $e_{0} \in E$ with $\widetilde{x}_{f(e)} \in U$ for all $e \geq e_{0}$. Now, fix $t \in \mathbb{Z}^{+}$and $\varepsilon>0$. By continuity of the map $x \mapsto \rho_{z}^{t}(x)$, there exists $e_{0} \in E$ such that $\left|\rho_{z}^{t}(\widetilde{x})-\rho_{z}^{t}\left(\widetilde{x}_{f(e)}\right)\right| \leq \varepsilon$ and $\widetilde{t}_{f(e)} \geq t$ for all $e \geq e_{0}$ (cf. Willard [17, Thm. 11.8]). Then

$$
\rho_{z}^{t}(\widetilde{x}) \leq \rho_{z}^{t}\left(\widetilde{x}_{f(e)}\right)+\left|\rho_{z}^{t}(\widetilde{x})-\rho_{z}^{t}\left(\widetilde{x}_{f(e)}\right)\right| \leq \rho_{*}+\varepsilon_{f(e)}+\widetilde{\varepsilon}_{f(e)}+\varepsilon \text { for all } e \geq e_{0} .
$$


Now, for each $k \in \mathbb{Z}^{+}$we find $\bar{e} \in E$ with $f(\bar{e}) \geq k$. Then there is $e \in E$ with both $e \geq e_{0}$ and $e \geq \bar{e}$, and hence

$$
\rho_{z}^{t}(\widetilde{x}) \leq \rho_{*}+\varepsilon_{f(e)}+\widetilde{\varepsilon}_{f(e)}+\varepsilon .
$$

Since $f(e) \geq f(\bar{e}) \geq k$ and $\varepsilon_{k}, \widetilde{\varepsilon}_{k} \searrow 0$ for $k \rightarrow \infty$, it follows that $\rho_{t}^{z}(\widetilde{x}) \leq \rho_{*}+\varepsilon$. Since $\varepsilon$ and $t$ have been chosen arbitrarily, we conclude

$$
\rho_{z}^{t}(\widetilde{x}) \leq \rho_{*} \text { for all } t \geq 1
$$

and thus $\limsup _{t \rightarrow \infty} \rho_{z}^{t}(\widetilde{x}) \leq \rho_{*}$. Now, assume that $\liminf _{t \rightarrow \infty} \rho_{z}^{t}(\widetilde{x})<\rho_{*}$. This implies the existence of a sequence $t_{k} \rightarrow \infty$ such that $\lim _{k \rightarrow \infty} \rho_{z}^{t_{k}}(\widetilde{x})<\rho_{*}=$ $\min \Sigma_{\mathrm{UG}}(\mathcal{M})$, which is a contradiction. Therefore, $x_{*}:=\widetilde{x}$ has the desired properties. With the same arguments we can prove the assertion for $\rho^{*}$.

Step 2. It remains to show that $\Sigma_{\mathrm{UG}}^{z}(\mathcal{M})$ is an interval. In Step 1, we have shown that $\Sigma_{\mathrm{UG}}^{z}(\mathcal{M}) \subset\left[\rho_{*}, \rho^{*}\right]$. Thus, we have to show that $\left[\rho_{*}, \rho^{*}\right] \subset \Sigma_{\mathrm{UG}}^{z}(\mathcal{M})$. Since $\mathcal{M}$ is compact and connected, and $\rho_{z}^{t}: X \rightarrow \mathbb{R}$ is continuous for each $t \geq 1$, the sets $\rho_{z}^{t}(\mathcal{M})$ are compact intervals. Furthermore, using (7), for each $t \geq 1$ it holds that $\rho_{z}^{t}\left(x_{*}\right) \leq \rho_{*}$ and $\rho_{z}^{t}\left(x^{*}\right) \geq \rho^{*}$, which implies $\left[\rho_{*}, \rho^{*}\right] \subset \rho_{z}^{t}(\mathcal{M})$ for all $t \geq 1$. Since we can write

$$
\Sigma_{\mathrm{UG}}^{z}(\mathcal{M})=\bigcap_{T>0} \mathrm{cl} \bigcup_{t \geq T} \rho_{z}^{t}(\mathcal{M})
$$

by Lemma 2 , we conclude that $\left[\rho_{*}, \rho^{*}\right] \subset \Sigma_{\mathrm{UG}}^{z}(\mathcal{M})$.

Corollary 2 Under the assumptions of Theorem 1 it holds that

$$
\inf _{x \in \mathcal{M}} \limsup _{t \rightarrow \infty} \rho_{z}^{t}(x)=\inf _{x \in \mathcal{M}} \liminf _{t \rightarrow \infty} \rho_{z}^{t}(x)=\lim _{t \rightarrow \infty} \inf _{x \in \mathcal{M}} \rho_{z}^{t}(x)
$$

and

$$
\sup _{x \in \mathcal{M}} \limsup _{t \rightarrow \infty} \rho_{z}^{t}(x)=\sup _{x \in \mathcal{M}} \liminf _{t \rightarrow \infty} \rho_{z}^{t}(x)=\lim _{t \rightarrow \infty} \sup _{x \in \mathcal{M}} \rho_{z}^{t}(x) .
$$

Proof Let us prove [8]. The proof of (9) works analogously. Write

$$
\begin{aligned}
\alpha & :=\inf _{x \in \mathcal{M}} \limsup _{t \rightarrow \infty} \rho_{z}^{t}(x), \\
\beta & :=\inf _{x \in \mathcal{M}} \liminf _{t \rightarrow \infty} \rho_{z}^{t}(x), \\
\gamma & :=\lim _{t \rightarrow \infty} \inf _{x \in \mathcal{M}} \rho_{z}^{t}(x) .
\end{aligned}
$$

Existence of the limit in the definition of $\gamma$ follows from superadditivity of the function $f(t):=t \inf _{x \in \mathcal{M}} \rho_{z}^{t}(x)$, which is proved as follows:

$$
\begin{aligned}
f(t+s) & =\inf _{x \in \mathcal{M}}\left\langle(t+s) \rho^{t+s}(x), z\right\rangle=\inf _{x \in \mathcal{M}}\left\langle t \rho^{t}(x)+s \rho^{s}(\Phi(t, x)), z\right\rangle \\
& =\inf _{x \in \mathcal{M}}\left[\left\langle t \rho^{t}(x), z\right\rangle+\left\langle s \rho^{s}(\Phi(t, x)), z\right\rangle\right] \\
& \geq t \inf _{x \in \mathcal{M}}\left\langle\rho^{t}(x), z\right\rangle+s \inf _{x \in \mathcal{M}}\left\langle\rho^{s}(\Phi(t, x)), z\right\rangle \\
& \geq t \inf _{x \in \mathcal{M}} \rho_{z}^{t}(x)+s \inf _{x \in \mathcal{M}} \rho_{z}^{s}(x)=f(t)+f(s) .
\end{aligned}
$$


Note that the inequalities $\gamma \leq \beta$ and $\beta \leq \alpha$ are trivial. Hence, it suffices to show that $\alpha \leq \gamma$. To this end, we first show that $\gamma \in \Sigma_{\mathrm{UG}}^{z}(\mathcal{M})$. By compactness of $\mathcal{M}$ and continuity of $\rho_{z}^{t}$ it follows that for each $t \geq 1$ there is $x_{t} \in \mathcal{M}$ with $\inf _{x \in \mathcal{M}} \rho_{z}^{t}(x)=$ $\rho_{z}^{t}\left(x_{t}\right)$. Choose an arbitrary sequence $t_{k} \rightarrow \infty$. Then

$$
\gamma=\lim _{k \rightarrow \infty} \rho_{z}^{t_{k}}\left(x_{t_{k}}\right) \in \Sigma_{\mathrm{UG}}^{z}(\mathcal{M})
$$

Now, using Theorem 1 , we find

$$
\alpha=\inf _{x \in \mathcal{M}} \limsup _{t \rightarrow \infty} \rho_{z}^{t}(x) \leq \lim _{t \rightarrow \infty} \rho_{z}^{t}\left(x_{*}\right)=\rho_{*} \leq \gamma,
$$

which completes the proof.

Remark 1 Note that in the proof of the preceding corollary we did not use that $\Sigma_{\mathrm{UG}}^{z}(\mathcal{M})$ is connected. Hence, looking at the proof of Theorem 1 , we see that we do not need the assumption that $\mathcal{M}$ is connected either for the validity of the identities (8) and 9 .

\section{The Morse Spectrum}

In this section, we introduce and study the Morse spectrum over a compact invariant set for a given growth rate. We will frequently assume that $X$ is a paracompact Hausdorff space and $\Phi: \mathbb{T}_{0}^{+} \times X \rightarrow X$ a semiflow on $X$. By $\mathcal{O}(X)$ we denote the family of all open covers of $X$.

Definition 3 Let $\mathcal{U} \in \mathcal{O}(X)$ and $x, y \in X, T \in \mathbb{T}^{+}$. A $(\mathcal{U}, T)$-chain $\zeta$ from $x$ to $y$ consists of an integer $n \geq 1$, a sequence of points $\left\{x=x_{0}, \ldots, x_{n}=y\right\} \subset X$, a sequence of times $\left\{T_{0}, \ldots, T_{n-1}\right\} \subset \mathbb{T}^{+}$with $T_{i} \geq T$ for $i=0, \ldots, n-1$, and a sequence of open sets $\left\{U_{1}, \ldots, U_{n}\right\} \subset \mathcal{U}$ such that for $i=0, \ldots, n-1$ we have

$$
\Phi\left(T_{i}, x_{i}\right), x_{i+1} \in U_{i+1} .
$$

The total length of $\zeta$ is defined by $\sum_{i=0}^{n-1} T_{i}$. We say that $\zeta$ is a chain in $\mathcal{M} \subset X$ if $\left\{x_{0}, \ldots, x_{n}\right\} \subset \mathcal{M}$. If $x_{0}=x_{n}, \zeta$ is called periodic. The chain $\zeta$ is called trivial if $x_{i+1}=\Phi\left(T_{i}, x_{i}\right)$ for $i=0, \ldots, n-1$.

Definition 4 For $\mathcal{U} \in \mathcal{O}(X), Y \subset X$ and $T \in \mathbb{T}^{+}$we define the $(\mathcal{U}, T)$-chain reachable set from $Y$ by

$$
\Omega(Y, \mathcal{U}, T):=\{x \in X: \exists y \in Y \text { and a }(\mathcal{U}, T) \text {-chain from } y \text { to } x\} .
$$

The $\Omega$-limit set of a subset $Y \subset X$ is defined by

$$
\Omega(Y):=\bigcap_{\substack{\mathcal{U} \in \mathcal{O}(X), T \in \mathbb{T}^{+}}} \Omega(Y, \mathcal{U}, T) .
$$

For $x \in X$ we write $\Omega(x):=\Omega(\{x\})$ and we define the relation

$$
x \preceq y: \Leftrightarrow y \in \Omega(x) .
$$


The next proposition can be found in Patrão \& San Martin [11, Prop. 3.3] (here the assumption of paracompactness of $X$ is essential, since this assumption guarantees that $\mathcal{O}(X)$ is an admissible family in the sense of [1].)

Proposition 4 The relation $\preceq$ is transitive, closed and $\Phi$-invariant. Here, "closed" means that for convergent sequences $x_{n}$ and $y_{n}$ in $X$ with $x_{n} \preceq y_{n}$ for all $n$, it holds that $\lim _{n \rightarrow \infty} x_{n} \preceq \lim _{n \rightarrow \infty} y_{n}$. " $\Phi$-invariant" means that $x \preceq y$ implies $\Phi(t, x) \preceq \Phi(s, y)$ for all $s, t \geq 0$.

Definition 5 We define the relation

$$
x \sim y: \Leftrightarrow x \preceq y \text { and } y \preceq x .
$$

A point $x \in X$ is called chain recurrent if $x \sim x$. The set $\mathcal{R}$ of all chain recurrent points is called the chain recurrent set. The restriction of $\sim$ to $\mathcal{R}$ is clearly an equivalence relation. A subset $Y \subset X$ is called chain transitive if for all $x, y \in Y$ it holds that $x \sim y$. An equivalence class of $\sim$ is called a chain recurrent component.

Remark 2 For a flow $\Phi: \mathbb{R} \times M \rightarrow M$ on a compact metric space $M$, the chain recurrent components are the connected components of the chain recurrent set (cf. Colonius \& Kliemann [3, Thm. B.2.22]).

Definition 6 Let $\rho: \mathbb{T}^{+} \times X \rightarrow \mathbb{R}^{m}$ be a growth rate for $\Phi$. We define the growth rate of a $(\mathcal{U}, T)$-chain $\zeta$ with times $T_{0}, \ldots, T_{n-1} \geq T$ and points $x_{0}, \ldots, x_{n} \in X$ by

$$
\rho(\zeta):=\left(\sum_{i=0}^{n-1} T_{i}\right)^{-1} \sum_{i=0}^{n-1} T_{i} \rho^{T_{i}}\left(x_{i}\right) .
$$

For $z \in S^{m-1}$ we also define the $z$-related growth rate of $\zeta$ by

$$
\rho_{z}(\zeta):=\left(\sum_{i=0}^{n-1} T_{i}\right)^{-1} \sum_{i=0}^{n-1} T_{i} \rho_{z}^{T_{i}}\left(x_{i}\right)=\langle\rho(\zeta), z\rangle
$$

Lemma 5 Let $\rho: \mathbb{T}^{+} \times X \rightarrow \mathbb{R}^{m}$ be a growth rate for $\Phi$, and $\zeta$ a $(\mathcal{U}, T)$-chain with times $T_{0}, \ldots, T_{n-1} \geq T$ and points $x_{0}, \ldots, x_{n} \in X$. Then for each $z \in S^{m-1}$ we have

$$
\min _{i=0, \ldots, n-1} \rho_{z}^{T_{i}}\left(x_{i}\right) \leq \rho_{z}(\zeta) \leq \max _{i=0, \ldots, n-1} \rho_{z}^{T_{i}}\left(x_{i}\right)
$$

Proof Let $\bar{T}:=T_{0}+\cdots+T_{n-1}$. The assertion for the maximum follows by

$$
\begin{aligned}
\rho_{z}(\zeta) & =\bar{T}^{-1}\left[T_{0} \rho_{z}^{T_{0}}\left(x_{0}\right)+\cdots+T_{n-1} \rho_{z}^{T_{n-1}}\left(x_{n-1}\right)\right] \\
& \leq \frac{T_{0}}{\bar{T}} \max _{i=0, \ldots, n-1} \rho_{z}^{T_{i}}\left(x_{i}\right)+\cdots+\frac{T_{n-1}}{\bar{T}} \max _{i=0, \ldots, n-1} \rho_{z}^{T_{i}}\left(x_{i}\right) \\
& =\max _{i=0, \ldots, n-1} \rho_{z}^{T_{i}}\left(x_{i}\right),
\end{aligned}
$$

and analogously for the minimum. 
Definition 7 Let $\xi$ be a $(\mathcal{U}, T)$-chain in $X$, given by points $\left\{x=x_{0}, \ldots, x_{n}=y\right\} \subset X$, times $\left\{T_{0}, \ldots, T_{n-1}\right\} \subset \mathbb{T}^{+}$and open sets $\left\{U_{1}, \ldots, U_{n}\right\} \subset \mathcal{U}$, and let $\zeta$ be another $(\mathcal{U}, T)$-chain given by points $\left\{y=z_{0}, \ldots, z_{m}\right\} \subset X$, times $\left\{S_{0}, \ldots, S_{m-1}\right\} \subset \mathbb{T}^{+}$and open sets $\left\{V_{1}, \ldots, V_{m}\right\} \subset \mathcal{U}$. We define the concatenated $(\mathcal{U}, T)$-chain $\zeta \circ \xi$ as the chain with points $\left\{p_{0}, \ldots, p_{n+m}\right\} \subset X$, times $\left\{R_{0}, \ldots, R_{n+m-1}\right\} \subset \mathbb{T}^{+}$and open sets $\left\{W_{1}, \ldots, W_{n+m}\right\} \subset \mathcal{U}$, given by

$$
\begin{gathered}
p_{i}:=\left\{\begin{array}{cc}
x_{i} & i=0, \ldots, n \\
z_{i-n} & i=n+1, \ldots, n+m
\end{array}, \quad R_{i}:=\left\{\begin{array}{cc}
T_{i} & i=0, \ldots, n-1 \\
S_{i-n} & i=n, \ldots, n+m-1
\end{array}\right.\right. \\
W_{i}:=\left\{\begin{array}{cc}
U_{i} & i=1, \ldots, n \\
V_{i-n} & i=n+1, \ldots, n+m
\end{array}\right.
\end{gathered}
$$

The proof of the next lemma follows by an easy computation which can be seen in Colonius et al. 11, Lem. 2.5].

Lemma 6 Let $\xi, \zeta$ be $(\mathcal{U}, T)$-chains in $X$ of total lengths $\sigma$ and $\tau$, respectively, such that the initial point of $\zeta$ coincides with the endpoint of $\xi$. Then for the concatenated chain $\zeta \circ \xi$ we have

$$
\rho(\zeta \circ \xi)=\frac{\sigma}{\sigma+\tau} \rho(\xi)+\frac{\tau}{\sigma+\tau} \rho(\zeta) .
$$

In the rest of this section, $\rho: \mathbb{T}^{+} \times X \rightarrow \mathbb{R}^{m}$ is a fixed growth rate for $\Phi$, and $\mathcal{M} \subset X$ is a compact $\Phi$-invariant set.

Definition 8 For each $\mathcal{U} \in \mathcal{O}(X), T \in \mathbb{T}^{+}$and $z \in S^{m-1}$ we define the sets

$$
\begin{aligned}
& \Sigma_{\mathrm{Mo}, \Phi, \rho}(\mathcal{M} ; \mathcal{U}, T):=\{\rho(\zeta): \zeta \text { is a }(\mathcal{U}, T) \text {-chain in } \mathcal{M}\} \\
& \Sigma_{\mathrm{Mo}, \Phi, \rho}^{z}(\mathcal{M} ; \mathcal{U}, T):=\left\{\rho_{z}(\zeta): \zeta \text { is a }(\mathcal{U}, T) \text {-chain in } \mathcal{M}\right\} .
\end{aligned}
$$

Then the Morse spectrum over $\mathcal{M}$ is defined by

$$
\Sigma_{\mathrm{Mo}, \Phi, \rho}(\mathcal{M}):=\bigcap_{\substack{\mathcal{U} \in \mathcal{O}(X), T \in \mathbb{T}^{+}}} \operatorname{cl} \Sigma_{\mathrm{Mo}, \Phi, \rho}(\mathcal{M} ; \mathcal{U}, T) .
$$

For each $z \in S^{m-1}$ the $z$-related Morse spectrum over $\mathcal{M}$ is defined by

$$
\Sigma_{\mathrm{Mo}, \Phi, \rho}^{z}(\mathcal{M}):=\bigcap_{\substack{\mathcal{U} \in \mathcal{O}(X), T \in \mathbb{T}^{+}}} \operatorname{cl} \Sigma_{\mathrm{Mo}, \Phi, \rho}^{z}(\mathcal{M} ; \mathcal{U}, T) .
$$

If it is clear from the context what $\Phi$ and $\rho$ are, we omit the corresponding indices.

The next proposition summarizes some elementary properties of the Morse spectrum.

Proposition 5 The following statements hold:

1. $\Sigma_{\mathrm{UG}}(\mathcal{M}) \subset \Sigma_{\mathrm{Mo}}(\mathcal{M})$ and $\Sigma_{\mathrm{UG}}^{z}(\mathcal{M}) \subset \Sigma_{\mathrm{Mo}}^{z}(\mathcal{M})$.

2. $\left\langle\Sigma_{\mathrm{Mo}}(\mathcal{M}), z\right\rangle \subset \Sigma_{\mathrm{Mo}}^{z}(\mathcal{M})$.

3. The sets $\Sigma_{\mathrm{Mo}}(\mathcal{M})$ and $\Sigma_{\mathrm{Mo}}^{z}(\mathcal{M})$ are compact.

Proof 
1. Let $\lambda \in \Sigma_{\mathrm{UG}}(\mathcal{M})$, i.e., $\lambda=\lim _{k \rightarrow \infty} \rho^{t_{k}}\left(x_{k}\right)$ with $x_{k} \in \mathcal{M}$ and $t_{k} \in \mathbb{T}^{+}, t_{k} \rightarrow \infty$. Fix $\mathcal{U} \in \mathcal{O}(X)$ and $T \in \mathbb{T}^{+}$. We have to show that $\lambda \in \operatorname{cl} \Sigma_{\mathrm{Mo}}(\mathcal{M} ; \mathcal{U}, T)$. To this end, define a sequence $\zeta_{k}$ of $(\mathcal{U}, T)$-chains in $\mathcal{M}$ as follows: let $k_{0} \in \mathbb{Z}^{+}$be chosen such that $t_{k} \geq T$ for all $k \geq k_{0}$. Then, for each $k \in \mathbb{Z}^{+}$, let $\zeta_{k}$ be the trivial chain consisting of points $\left\{x_{k+k_{0}}, \Phi\left(t_{k+k_{0}}, x_{k+k_{0}}\right)\right\}$, time(s) $\left\{t_{k+k_{0}}\right\}$, and open set(s) $\left\{U_{k}\right\}$, where $U_{k} \in \mathcal{U}$ is chosen such that $\Phi\left(t_{k+k_{0}}, x_{k+k_{0}}\right) \in U_{k}$. Then $\zeta_{k}$ clearly is a $(\mathcal{U}, T)$-chain in $\mathcal{M}$ for each $k \in \mathbb{Z}^{+}$and $\rho\left(\zeta_{k}\right)=\rho^{t_{k+k_{0}}}\left(x_{k+k_{0}}\right)$, which implies

$$
\lambda=\lim _{k \rightarrow \infty} \rho^{t_{k}}\left(x_{k}\right)=\lim _{k \rightarrow \infty} \rho\left(\zeta_{k}\right) \in \operatorname{cl} \Sigma_{\mathrm{Mo}}(\mathcal{M} ; \mathcal{U}, T)
$$

The corresponding assertion for $\Sigma_{\mathrm{UG}}^{z}(\mathcal{M})$ is proved analogously.

2. This follows from continuity of the function $x \mapsto\langle x, z\rangle$ and the obvious relation $\left\langle\Sigma_{\mathrm{Mo}}(\mathcal{M} ; \mathcal{U}, T), z\right\rangle=\Sigma_{\text {Mo }}^{z}(\mathcal{M} ; \mathcal{U}, T)$, which holds for all $\mathcal{U} \in \mathcal{O}(X), T \in \mathbb{T}^{+}$.

3. Closedness of $\Sigma_{\mathrm{Mo}}(\mathcal{M})$ and $\Sigma_{\mathrm{Mo}}^{z}(\mathcal{M})$ follows immediately from the definitions. Boundedness follows from boundedness of $\rho$ on $\mathbb{T}_{\geq 1} \times X$.

If we additionally assume that the set $\mathcal{M}$ is connected and that the restriction of $\Phi$ to $\mathcal{M}$ is chain transitive, we can say more about the Morse spectrum and its relations to the uniform growth spectrum and the Lyapunov spectrum. To prove the corresponding results, we need the following two lemmas.

Lemma 7 Assume that the restriction of $\Phi$ to $\mathcal{M}$ is chain transitive and fix $\mathcal{U} \in \mathcal{O}(X)$ and $T \in \mathbb{T}^{+}$. Then there exists $\bar{T}(\mathcal{U}, T) \in \mathbb{T}^{+}$such that for all $x, y \in \mathcal{M}$ there is a $(\mathcal{U}, T)$-chain from $x$ to $y$ with total length $\leq \bar{T}(\mathcal{U}, T)$.

Proof By chain transitivity, for all $x, y \in \mathcal{M}$ there is a $(\mathcal{U}, T)$-chain in $\mathcal{M}$ from $x$ to $y$. Fix $z \in \mathcal{M}$. By compactness of $\mathcal{M}$ and continuity of $\Phi$, there are finitely many $(\mathcal{U}, T)$-chains connecting every $x \in \mathcal{M}$ to $z$, if we do not distinguish between chains which only differ in their initial points. Similarly, (modulo their endpoints) there are only finitely many $(\mathcal{U}, T)$-chains connecting $z$ with arbitrary $y \in \mathcal{M}$. Hence, there are finitely many $(\mathcal{U}, T)$-chains connecting all points in $\mathcal{M}$. The maximum of their total lengths is the desired upper bound $\bar{T}(\mathcal{U}, T)$.

The next lemma says that we only have to consider periodic chains to obtain the Morse spectrum. The proof is essentially the same as that of Colonius et al. 1 , Prop. 2.6].

Lemma 8 Assume that the restriction of $\Phi$ to $\mathcal{M}$ is chain transitive. For each $\mathcal{U} \in$ $\mathcal{O}(X)$ and $T \in \mathbb{T}^{+}$let

$$
\Sigma_{\mathrm{Mo}, \operatorname{Per}}(\mathcal{M} ; \mathcal{U}, T):=\{\rho(\zeta): \zeta \text { is a periodic }(\mathcal{U}, T) \text {-chain in } \mathcal{M}\}
$$

Then it holds that

$$
\Sigma_{\mathrm{Mo}}(\mathcal{M})=\bigcap_{\substack{\mathcal{U} \in \mathcal{O}(X), T \in \mathbb{T}^{+}}} \operatorname{cl} \Sigma_{\mathrm{Mo}, \operatorname{Per}}(\mathcal{M} ; \mathcal{U}, T)
$$

Proof Let $\lambda \in \Sigma_{\mathrm{Mo}}(\mathcal{M})$ and fix $\mathcal{U} \in \mathcal{O}(X)$ and $T \in \mathbb{T}_{\geq 1}$. It suffices to prove that for every $\delta>0$ there exists a periodic $(\mathcal{U}, T)$-chain $\zeta^{\prime}$ with $\left\|\lambda-\rho\left(\zeta^{\prime}\right)\right\|<\delta$. By Lemma 7 . there exists $\bar{T}(\mathcal{U}, T) \in \mathbb{T}^{+}$such that for all $x, y \in \mathcal{M}$ there is a $(\mathcal{U}, T)$-chain from $x$ to $y$ with total time $\leq \bar{T}(\mathcal{U}, T)$. For $S>T$ choose a $(\mathcal{U}, S)$-chain $\zeta$ with $\|\lambda-\rho(\zeta)\|<\delta / 2$ 
given by points $\left\{x_{0}, \ldots, x_{n}\right\} \subset \mathcal{M}$, times $\left\{S_{0}, \ldots, S_{n-1}\right\} \subset \mathbb{T}^{+}$with $S_{i} \geq S$ for $i=0, \ldots, n-1$, and open sets $\left\{U_{1}, \ldots, U_{n}\right\} \subset \mathcal{U}$ with total time $\sigma=\sum_{i=0}^{n-1} S_{i}$. Concatenate $\zeta$ with a $(\mathcal{U}, T)$-chain $\xi$ from $x_{n}$ to $x_{0}$, given by points $\left\{x_{n}=y_{0}, \ldots, y_{m}=\right.$ $\left.x_{0}\right\} \subset \mathcal{M}$, times $\left\{T_{0}, \ldots, T_{m-1}\right\} \subset \mathbb{T}^{+}$with $T_{i} \geq T$ for $i=0, \ldots, m-1$, and open sets $\left\{\widetilde{U}_{1}, \ldots, \widetilde{U}_{m}\right\} \subset \mathcal{U}$ with total time $\tau=\sum_{i=0}^{m-1} T_{i} \leq \bar{T}(\mathcal{U}, T)$. The periodic $(\mathcal{U}, T)$-chain $\zeta^{\prime}:=\xi \circ \zeta$ has the desired approximation property: Since the chain $\xi$ depends on $\zeta$, also $\tau$ depends on $\zeta$. However, the total length of $\xi$ is bounded. Moreover, Lemma 6 implies

$$
\begin{aligned}
\|\rho(\zeta)-\rho(\xi \circ \zeta)\| & =\left\|\rho(\zeta)-\frac{\sigma}{\sigma+\tau} \rho(\zeta)-\frac{\tau}{\sigma+\tau} \rho(\xi)\right\| \\
& \leq\left[1-\frac{\sigma}{\sigma+\tau}\right]\|\rho(\zeta)\|+\frac{\tau}{\sigma+\tau}\|\rho(\xi)\| .
\end{aligned}
$$

Since $T \geq 1$ (which we could assume without loss of generality), we have $\max \{\|\rho(\zeta)\|,\|\rho(\xi)\|\} \leq M$. Since $\tau$ remains bounded for $S \rightarrow \infty$, the right-hand side tends to 0 as $S \rightarrow \infty$.

Theorem 2 If $\mathcal{M}$ is connected and the restriction of $\Phi$ to $\mathcal{M}$ is chain transitive, then the following statements hold:

1. $\Sigma_{\mathrm{Mo}}^{z}(\mathcal{M})=\Sigma_{\mathrm{UG}}^{z}(\mathcal{M})$ for each $z \in S^{m-1}$.

2. $\left\langle\Sigma_{\mathrm{Mo}}(\mathcal{M}), z\right\rangle=\Sigma_{\mathrm{Mo}}^{z}(\mathcal{M})$ for each $z \in S^{m-1}$.

3. $\Sigma_{\mathrm{Mo}}(\mathcal{M})$ is a convex set.

4. Every exposed point $p \in \Sigma_{\mathrm{Mo}}(\mathcal{M})$ is the Lyapunov exponent of some point $x \in \mathcal{M}$, i.e., there exists $x \in \mathcal{M}$ with $\lim _{t \rightarrow \infty} \rho^{t}(x)=p$. Hence, $\Sigma_{\mathrm{Mo}}(\mathcal{M})$ is the closed convex hull of the Lyapunov spectrum $\Sigma_{\mathrm{Ly}}(\mathcal{M})$, and the convex hull of the uniform growth spectrum $\Sigma_{\mathrm{UG}}(\mathcal{M})$.

Proof

1. By Proposition $5(3)$ and Proposition 3 both $\Sigma_{\mathrm{UG}}^{z}(\mathcal{M})$ and $\Sigma_{\mathrm{Mo}}^{z}(\mathcal{M})$ are compact, hence they have minimal and maximal elements. Moreover, by Proposition 5 (1), the inclusion $\Sigma_{\mathrm{UG}}^{z}(\mathcal{M}) \subset \Sigma_{\mathrm{Mo}}^{z}(\mathcal{M})$ holds. By Theorem 1, $\Sigma_{\mathrm{UG}}^{z}(\mathcal{M})$ is an interval. Hence, it suffices to show that $\min \Sigma_{\mathrm{Mo}}^{z}(\mathcal{M}) \geq \min \Sigma_{\mathrm{UG}}^{z}(\mathcal{M})$ and $\max \Sigma_{\mathrm{Mo}}^{z}(\mathcal{M}) \leq$ $\max \Sigma_{\mathrm{UG}}^{z}(\mathcal{M})$. Without loss of generality, we only prove the first inequality. To this end, choose an arbitrary sequence $\mathcal{U}_{k} \in \mathcal{O}(X)$ and a sequence of times $T_{k} \in \mathbb{T}^{+}$ with $T_{k} \rightarrow \infty$. Since $\min \Sigma_{\text {Mo }}^{z}(\mathcal{M})=: \lambda \in \Sigma_{\text {Mo }}^{z}(\mathcal{M})$, we find for each $k$ a $\left(\mathcal{U}_{k}, T_{k}\right)$ chain $\zeta_{k} \subset \mathcal{M}$ with $\left|\lambda-\rho_{z}\left(\zeta_{k}\right)\right| \leq 1 / 2^{k}$. By Lemma 5 , we can take a point $x_{k}$ in the chain $\zeta_{k}$ and a time $t_{k} \geq T_{k}$ such that $\rho_{z}^{t_{k}}\left(x_{k}\right) \leq \rho_{z}\left(\zeta_{k}\right)$. Then there is a convergent subsequence $\rho_{z}^{t_{k_{l}}}\left(x_{k_{l}}\right)$ with limit $\mu=\lim _{l \rightarrow \infty} \rho_{z}^{t_{k_{l}}}\left(x_{k_{l}}\right) \in \Sigma_{\mathrm{UG}}^{z}(\mathcal{M})$. To complete the proof, it suffices to show that $\mu \leq \lambda$, which is proved by

$$
\mu-\lambda=\lim _{l \rightarrow \infty}\left(\rho_{z}^{t_{k_{l}}}\left(x_{k_{l}}\right)-\lambda\right) \leq \limsup _{l \rightarrow \infty}\left(\rho_{z}\left(\zeta_{k_{l}}\right)-\lambda\right) \leq \limsup _{l \rightarrow \infty} \frac{1}{2^{k_{l}}}=0 .
$$

2. For the inclusion " $\subset$ ", see Proposition 5 (2). The converse inclusion follows from statement (1), Proposition 2 and Proposition 5 (1):

$$
\Sigma_{\mathrm{Mo}}^{z}(\mathcal{M})=\Sigma_{\mathrm{UG}}^{z}(\mathcal{M})=\left\langle\Sigma_{\mathrm{UG}}(\mathcal{M}), z\right\rangle \subset\left\langle\Sigma_{\mathrm{Mo}}(\mathcal{M}), z\right\rangle .
$$


3. We adapt the proof of Colonius et al. [1, Thm. 2.7]: Let $\lambda$ be an element of the convex hull of $\Sigma_{\mathrm{Mo}}(\mathcal{M})$. It suffices to show that for each $\delta>0, \mathcal{U} \in \mathcal{O}(X)$ and $T \in \mathbb{T}^{+}$there is a $(\mathcal{U}, T)$-chain $\zeta$ in $\mathcal{M}$ with $\|\rho(\zeta)-\lambda\| \leq \delta$. So let $\lambda=\sum_{i=0}^{n} \alpha_{i} \lambda_{i}$ with $\alpha_{i}>0$ and $\sum_{i=0}^{n} \alpha_{i}=1, \lambda_{i} \in \Sigma_{\mathrm{Mo}}(\mathcal{M})$. By Lemma 8 , there are periodic $(\mathcal{U}, T)$-chains $\zeta_{i}, i=0,1, \ldots, n$, in $\mathcal{M}$ with

$$
\left\|\rho\left(\zeta_{i}\right)-\lambda_{i}\right\| \leq \delta \text { for } i=0,1, \ldots, n .
$$

Denote the initial (and final) point of $\zeta_{i}$ by $x_{i}$. By chain transitivity, there are $(\mathcal{U}, T)$-chains $\xi_{i}$ from $x_{i}$ to $x_{i+1}$ and $\xi_{n}$ from $x_{n}$ to $x_{0}$. For $k \in \mathbb{Z}^{+}$, let $\zeta_{i, k}$ be the $k$-fold concatenation of $\zeta_{i}$. Then for arbitrary $k_{1}, \ldots, k_{n} \in \mathbb{Z}^{+}$the concatenation $\zeta\left(k_{1}, \ldots, k_{n}\right):=\xi_{n} \circ \zeta_{n, k_{n}} \circ \cdots \circ \xi_{1} \circ \zeta_{1, k_{1}}$ is a periodic $(\mathcal{U}, T)$-chain in $\mathcal{M}$. From Lemma 6 it follows that we can choose $k_{1}, \ldots, k_{n}$ sufficiently large such that $\left\|\rho\left(\zeta\left(k_{1}, \ldots, k_{n}\right)\right)-\lambda\right\| \leq \delta$, which finishes the proof.

4. Let $p \in \Sigma_{\mathrm{Mo}}(\mathcal{M})$ be an exposed point. There exists a supporting hyperplane $H$ given by a vector $z \in S^{m-1}$, i.e., $H=\left\{x \in \mathbb{R}^{m}:\langle x, z\rangle=\langle p, z\rangle\right\}$ and

$$
\Sigma_{\mathrm{Mo}}(\mathcal{M}) \backslash\{p\} \subset\left\{x \in \mathbb{R}^{m}:\langle x, z\rangle<\langle p, z\rangle\right\} .
$$

By statements $(1)$ and $(2)$, it holds that $\left\langle\Sigma_{\mathrm{Mo}}(\mathcal{M}), z\right\rangle=\left\langle\Sigma_{\mathrm{UG}}(\mathcal{M}), z\right\rangle$, and by 10p, $\langle p, z\rangle$ is the right boundary point of $\left\langle\Sigma_{\mathrm{Mo}}(\mathcal{M}), z\right\rangle$. Theorem 1 implies the existence of $x^{*} \in \mathcal{M}$ such that $\lim _{t \rightarrow \infty}\left\langle\rho^{t}\left(x^{*}\right), z\right\rangle=\langle p, z\rangle$. It remains to show that $\lim _{k \rightarrow \infty} \rho^{t_{k}}\left(x^{*}\right)=p$ for every sequence $t_{k} \rightarrow \infty$. To this end, assume that there is a limit point $\widetilde{p} \neq p$, i.e., $\widetilde{p}=\lim _{k \rightarrow \infty} \rho^{t_{k}}\left(x^{*}\right)$ for some sequence $t_{k} \rightarrow \infty$. Then $\langle\widetilde{p}, z\rangle \in\left\langle\Sigma_{\mathrm{Mo}}(\mathcal{M}), z\right\rangle$, and hence $\langle\widetilde{p}, z\rangle<\langle p, z\rangle$, which is a contradiction.

Proof This follows from the fact that the Morse spectrum is the convex hull of the uniform growth spectrum (both in continuous- and discrete time) and Proposition 1.

Remark 3 For a compact, connected and chain transitive set $\mathcal{M}$ we have found the following relations for the different spectral sets associated with a growth rate:

$$
\Sigma_{\mathrm{Ly}}(\mathcal{M}) \subset \Sigma_{\mathrm{UG}}(\mathcal{M}) \subset \operatorname{clco} \Sigma_{\mathrm{Ly}}(\mathcal{M})=\operatorname{co} \Sigma_{\mathrm{UG}}(\mathcal{M})=\Sigma_{\mathrm{Mo}}(\mathcal{M}) .
$$

Instead of assuming paracompactness of $X$ and taking the family $\mathcal{O}(X)$ of all open covers of the space $X$ in the definition of the Morse spectrum, it would also be possible to work with admissible families of open covers in the sense of Patrão \& San Martin 11. However, as indicated in San Martin \& Seco 8, in this more general setting we would still find that for $\mathcal{M}$ being connected, the Morse spectrum is the closed convex hull of the Lyapunov spectrum, and hence independent of the chosen family of covers. In fact, that is the reason why we chose not to work in this general setting.

Remark 4 We defined the Morse spectrum in the same way as this is done in San Martin \& Seco 8] for vector-valued cocycles (cf. also Example 4). In the special cases of Examples 1 and 3 our definition gives the same set as the usual definition via $(\varepsilon, T)$ chains in metric spaces. In Stender [15], a somewhat different definition of the Morse spectrum is used which also coincides with the usual one in the corresponding special cases.

We end this section by describing a transformation that preserves all the spectral sets which we introduced for growth rates (cf. San Martin \& Seco [8, Cor. 3.5]). 
Proposition 6 Consider two semiflows $\Phi_{1}: \mathbb{T}_{0}^{+} \times X_{1} \rightarrow X_{1}$ and $\Phi_{2}: \mathbb{T}_{0}^{+} \times X_{2} \rightarrow X_{2}$ with the same time set. Let $\rho_{1}$ and $\rho_{2}$ be corresponding growth rates, which are related by

$$
\rho_{2}^{t}(\pi(x))=L\left(\rho_{1}^{t}(x)\right) \text { for all } t \in \mathbb{T}^{+}, x \in X_{1},
$$

where $\pi: X_{1} \rightarrow X_{2}$ is a continuous map and $L: \mathbb{R}^{m} \rightarrow \mathbb{R}^{m}$ a linear automorphism. If $\mathcal{M} \subset X_{1}$ is a compact $\Phi_{1}$-invariant set such that $\pi(\mathcal{M}) \subset X_{2}$ is $\Phi_{2}$-invariant, then

$$
L \Sigma_{\mathrm{Ly}, \Phi_{1}, \rho_{1}}(\mathcal{M})=\Sigma_{\mathrm{Ly}, \Phi_{2}, \rho_{2}}(\pi(\mathcal{M})),
$$

and the same holds for the uniform growth spectra. If $\mathcal{M}$ and $\pi(\mathcal{M})$ are additionally connected and chain transitive, the same holds for the Morse spectra.

Proof Let $x \in \mathcal{M}$ and assume that the Lyapunov exponent $\lambda(x)=\lim _{t \rightarrow \infty} \rho_{1}^{t}(x)$ exists. Then $\rho_{2}^{t}(\pi(x))=L\left(\rho_{1}^{t}(x)\right)$ converges to $L(\lambda(x))=\lambda(\pi(x))$ for $t \rightarrow \infty$. Hence, $L \Sigma_{\mathrm{Ly}, \Phi_{1}, \rho_{1}}(\mathcal{M}) \subset \Sigma_{\mathrm{Ly}, \Phi_{2}, \rho_{2}}(\pi(\mathcal{M}))$. The converse inclusion holds by invertibility of $L$. Now, let $\lambda$ be an element of $\Sigma_{\mathrm{UG}, \Phi_{1}, \rho_{1}}(\mathcal{M})$, i.e., $\lambda=\lim _{k \rightarrow \infty} \rho_{1}^{t_{k}}\left(x_{k}\right)$ with $x_{k} \in \mathcal{M}$ and $t_{k} \rightarrow \infty$. Then $\rho_{2}^{t_{k}}\left(\pi\left(x_{k}\right)\right)=L\left(\rho_{1}^{t_{k}}\left(x_{k}\right)\right) \rightarrow L(\lambda) \in \Sigma_{\mathrm{UG}, \Phi_{2}, \rho_{2}}(\pi(\mathcal{M}))$. The other inclusion again follows by invertibility of $L$. If $\mathcal{M}$ and $\pi(\mathcal{M})$ are connected and chain transitive, they are the closed convex hulls of $\Sigma_{\mathrm{Ly}, \Phi_{1}, \rho_{1}}(\mathcal{M})$ and $\Sigma_{\mathrm{Ly}, \Phi_{2}, \rho_{2}}(\pi(\mathcal{M}))$, respectively. By linearity and invertibility of $L$, this implies $L \Sigma_{\mathrm{Mo}, \Phi_{1}, \rho_{1}}(\mathcal{M})=\Sigma_{\mathrm{Mo}, \Phi_{2}, \rho_{2}}(\pi(\mathcal{M}))$.

Remark 5 For two linear flows on vector bundles and the growth rates from Example 1 (finite-time Lyapunov exponents), a transformation as in the preceding proposition, e.g., is given if there exists a cohomology between the two flows, i.e., a fiber preserving homeomorphism which is linear on the fibers and conjugates the two flows.

\section{The Meaning of Limit Sets for Growth Rates}

In this section, we show that for the analysis of the long-time behavior of growth rates it is sufficient to consider initial values contained in $\omega$-limit sets. Consider again a semiflow $\Phi: \mathbb{T}_{0}^{+} \times X \rightarrow X$ on a paracompact Hausdorff space $X$. For any subset $Y \subset X$ we define the $\omega$-limit set of $Y$ by

$$
\omega(Y):=\bigcap_{t \geq 0} \operatorname{cl} \bigcup_{s \geq t} \Phi(s, Y) .
$$

For $Y=\{x\}$, we write $\omega(x):=\omega(\{x\})$. Again, we denote by $\mathcal{O}(X)$ the family of all open covers of $X$.

The following proposition is taken from Conley [4, II.4.1.D]. Its proof is based on elementary topological arguments.

Proposition 7 Let $\mathcal{M} \subset X$ be a compact $\Phi$-invariant set. If $x \in \mathcal{M}$, then $\omega(x)$ is a nonempty compact invariant set in $\mathcal{M}$. If $\mathbb{T}=\mathbb{R}$, then $\omega(x)$ is connected. Furthermore, if $U$ is any neighborhood of $\omega(x)$, then there is $t \in \mathbb{T}^{+}$such that $\Phi(t, x) \in U$.

Proposition 8 In addition to the assumptions of the preceding proposition, let $\mathcal{U} \in$ $\mathcal{O}(X)$. Then there are finitely many sets $U_{1}, \ldots, U_{k} \in \mathcal{U}$ with $\omega(x) \subset U_{1} \cup \ldots \cup U_{k}$ and a time $T=T(\mathcal{U})$ such that for each $t \geq T$ there is $i \in\{1, \ldots, k\}$ with $\Phi(t, x) \in U_{i}$. 
Proof By Proposition 7, $\omega(x)$ is compact. Hence, $\omega(x)$ is covered by finitely many sets $U_{1}, \ldots, U_{k} \in \mathcal{U}$. Assume to the contrary that there is a strictly increasing sequence $t_{l} \rightarrow \infty$ such that $\Phi\left(t_{l}, x\right) \notin U_{i}$ for all $i \in\{1, \ldots, k\}$ and $l \in \mathbb{Z}^{+}$. Then on the one hand we have

$$
\omega(x)=\bigcap_{t \geq 0} \operatorname{cl} \bigcup_{s \geq t} \Phi(s,\{x\})=\bigcap_{l \in \mathbb{Z}^{+}} \mathrm{cl} \bigcup_{s \geq t_{l}} \Phi(s,\{x\}) \supset \bigcap_{l \in \mathbb{Z}^{+}} \operatorname{cl} \bigcup_{k \geq l} \Phi\left(t_{k},\{x\}\right),
$$

and on the other hand $\bigcap_{l \in \mathbb{Z}^{+}} \operatorname{cl} \bigcup_{k \geq l} \Phi\left(t_{k},\{x\}\right)$ is a nonempty subset of the closed set $X \backslash\left(U_{1} \cup \ldots \cup U_{k}\right)$, and hence disjoint from $\omega(x)$.

We will make use of the following lemma, which can be considered as an analogue of uniform continuity on compact sets. For the special case $M=X$, the lemma can be found in Patrão \& San Martin [11, Lem. 3.5], but for $M \neq X$ the proof works totally analogously, hence we will not repeat it here.

Lemma 9 Let $X, Y$ and $M$ be topological spaces, $Y$ compact, and $F: X \times Y \rightarrow M a$ continuous mapping. Let $\mathcal{U} \in \mathcal{O}(M)$. Then there is $\mathcal{Z} \in \mathcal{O}(X)$ such that the following holds: for arbitrary $y \in Y$ and $u, v \in Z$ for some $Z \in \mathcal{Z}$, there exists $U \in \mathcal{U}$ with $F(u, y), F(v, y) \in U$.

Lemma 10 Let $\rho: \mathbb{T}^{+} \times X \rightarrow \mathbb{R}^{m}$ be a growth rate for $\Phi$. Consider a trivial chain $\xi$ from $x$ to $\Phi(T, x)$ defined by

$$
x_{0}:=x, x_{1}:=\Phi\left(t_{0}, x_{0}\right), x_{2}:=\Phi\left(t_{1}, x_{1}\right), \ldots, x_{n}:=\Phi\left(t_{n-1}, x_{n-1}\right)=\Phi(T, x),
$$

where $T=\sum_{i=0}^{n-1} t_{i}$. Then we have $\rho(\xi)=\rho^{T}(x)$.

Proof We prove the lemma by induction over the length $n$ of the chains. For $n=1$ the assertion trivially holds. Assume that it holds for a fixed $n \in \mathbb{Z}^{+}$. Define $T_{0}:=\sum_{i=0}^{n-1} t_{i}$ and $T:=T_{0}+t_{n}$. Then we have

$$
\begin{aligned}
T \rho^{T}\left(x_{0}\right) & =T_{0} \rho^{T_{0}}\left(x_{0}\right)+t_{n} \rho^{t_{n}}\left(\Phi\left(T_{0}, x_{0}\right)\right) \\
& =T_{0} \cdot \frac{1}{\sum_{i=0}^{n-1} t_{i}} \sum_{i=0}^{n-1} t_{i} \rho^{t_{i}}\left(x_{i}\right)+t_{n} \rho^{t_{n}}\left(x_{n}\right)=\sum_{i=0}^{n} t_{i} \rho^{t_{i}}\left(x_{i}\right) .
\end{aligned}
$$

Dividing both sides of the equation by $T$ concludes the proof.

For open covers $\mathcal{U}, \mathcal{V} \in \mathcal{O}(X)$ we write $\mathcal{V} \leq 1 / 2 \mathcal{U}$ if for each two sets $V_{1}, V_{2} \in \mathcal{V}$ with $V_{1} \cap V_{2} \neq \emptyset$ there exists $U \in \mathcal{U}$ with $V_{1} \cup V_{2} \subset U$. The following lemma, for which paracompactness of $X$ is essential, is an easy consequence of a theorem of A. H. Stone which characterizes paracompactness in terms of open star-refinements (cf. Dugundji [5, Ch. VIII, Thm. 3.5]).

Lemma 11 For every $\mathcal{U} \in \mathcal{O}(X)$ there exists $\mathcal{V} \in \mathcal{O}(X)$ such that $\mathcal{V} \leq 1 / 2 \mathcal{U}$

The meaning of limit sets for the analysis of growth rates becomes clear in the following theorem, which is a generalization of Colonius et al. [1, Thm. 2.7] or Colonius \& Kliemann [3, Thm. 5.3.6]. For simplicity, we restrict ourselves to the continuous-time case. 
Theorem 3 Let $\mathcal{M} \subset X$ be a compact $\Phi$-invariant set and $\rho: \mathbb{R}^{+} \times X \rightarrow \mathbb{R}^{m}$ a growth rate for $\Phi$. If $\lim _{k \rightarrow \infty} \rho^{t_{k}}(x)$ exists for some $x \in \mathcal{M}$ and some sequence $t_{k} \rightarrow \infty$, then

$$
\lim _{k \rightarrow \infty} \rho^{t_{k}}(x) \in \Sigma_{M o}(\omega(x)) .
$$

Proof Let $\widetilde{x} \in \mathcal{M}$ and let $s_{k} \in \mathbb{R}^{+}$be a sequence with $s_{k} \rightarrow \infty$ such that $\lambda:=$ $\lim _{k \rightarrow \infty} \rho^{s_{k}}(\widetilde{x})$ exists. Fix $\mathcal{U} \in \mathcal{O}(X), T>1$ and $\delta>0$. It suffices to show that there exists a $(\mathcal{U}, T)$-chain $\xi$ in $\omega(\widetilde{x})$ with $\|\rho(\xi)-\lambda\| \leq \delta$.

Since $t \cdot \rho:[T, 2 T] \times X \rightarrow \mathbb{R}^{m},(t, x) \mapsto t \rho^{t}(x)$, is continuous, Lemma 9 implies the existence of an open cover $\mathcal{V} \in \mathcal{O}(X)$ such that if $a, b \in V$ for some $V \in \mathcal{V}$, then

$$
\left\|t \rho^{t}(a)-t \rho^{t}(b)\right\| \leq \frac{\delta}{2} \text { for all } t \in[T, 2 T] .
$$

By Lemma 11, we may assume that $\mathcal{V} \leq 1 / 2 \mathcal{U}$.

Since $\Phi:[0,2 T] \times X \rightarrow X$ is continuous, Lemma 9 implies the existence of an open cover $\mathcal{Z} \in \mathcal{O}(X)$ such that, for arbitrary $t \in[0,2 T]$ and $v, w \in Z$ for some $Z \in \mathcal{Z}$, we have that $\Phi(t, v), \Phi(t, w) \in V$ for some $V \in \mathcal{V}$. We may assume that $\mathcal{Z} \leq 1 / 2 \mathcal{V}$ (in fact, $\mathcal{Z} \leq \mathcal{V}$ will be sufficient.)

By Proposition 7 , the set $\omega(\widetilde{x})$ is compact. Hence, there are finitely many sets $Z_{1}, \ldots, Z_{l} \in \mathcal{Z}$ such that $\omega(\widetilde{x}) \subset \bigcup_{i=1}^{l} Z_{i}$. Furthermore, since $\mathcal{Z} \leq 1 / 2 \mathcal{V}$, there also exists a finite cover $\left\{V_{1}, \ldots, V_{l}\right\} \subset \mathcal{V}$ of $\omega(\widetilde{x})$ with

$$
Z_{j} \subset V_{j} \text { for } j=1, \ldots, l .
$$

By Proposition 8, there exists a time $S=S(\mathcal{Z})$ such that for any $t \geq S$ there is $j \in\{1, \ldots, l\}$ with $\Phi(t, \widetilde{x}) \in Z_{j}$. Let $x:=\Phi(S, \widetilde{x})$. Then for all $t>0$ there is $j \in\{1, \ldots, l\}$ with $\Phi(t, x) \in Z_{j}$, and by Lemma 1. for the sequence $t_{k}:=s_{k}-S \rightarrow \infty$ it holds that

$$
\lim _{k \rightarrow \infty} \rho^{t_{k}}(x)=\lambda \text {. }
$$

Hence, there exists $K \in \mathbb{Z}^{+}$such that

$$
\left\|\rho^{t_{k}}(x)-\lambda\right\| \leq \frac{\delta}{2} \text { for all } k \geq K .
$$

Choose $k$ large enough such that $T_{0}:=t_{k} \geq \max \left\{t_{K}, 2 T\right\}$. Since $T_{0}=n T+\widetilde{r}$ with $n \in \mathbb{Z}^{+}$and $\widetilde{r} \in[0, T)$, it holds that $T_{0}=(n-1) T+r$ with $r \in[T, 2 T)$. Since $T>1$, we have

$$
T_{0}>n
$$

Now define a trivial $(\mathcal{U}, T)$-chain $\widetilde{\xi}$ with times

$$
\tau_{0}:=\cdots:=\tau_{n-2}:=T, \quad \tau_{n-1}:=r
$$

and points

$$
x_{0}:=x, \quad x_{1}:=\Phi\left(T, x_{0}\right), \quad x_{2}:=\Phi\left(T, x_{1}\right), \quad \ldots, \quad x_{n}:=\Phi\left(r, x_{n-1}\right) .
$$

Lemma 10 implies that $\rho^{T_{0}}(x)=\rho(\widetilde{\xi})$. Now we have a chain $\widetilde{\xi}$ with

$$
\|\lambda-\rho(\widetilde{\xi})\| \leq \frac{\delta}{2}
$$


The chain $\widetilde{\xi}$ is not necessarily a chain in $\omega(x)=\omega(\widetilde{x})$. Thus, we have to construct an appropriate chain in $\omega(x)$.

For each $x_{j}, j=0, \ldots, n-1$, there exists $i(j) \in\{1, \ldots, l\}$ such that $x_{j} \in Z_{i(j)}$. Choose some $y_{j} \in Z_{i(j)} \cap \omega(x)$ (we may assume that this intersection is nonempty, since otherwise we could take a smaller subcover). By 12 we have $Z_{i(j)} \subset V_{i(j)}$ and, by choice of $\mathcal{Z}$, there exists a set $V^{j+1} \in \mathcal{V}$ with

$$
x_{j+1}=\Phi\left(\tau_{j}, x_{j}\right), \Phi\left(\tau_{j}, y_{j}\right) \in V^{j+1} .
$$

Applying the same construction to $x_{j+1}$ implies

$$
x_{j+1} \in V^{j+1} \cap V_{i(j+1)}, \Phi\left(\tau_{j}, y_{j}\right) \in V^{j+1} \text { and } y_{j+1} \in V_{i(j+1)} .
$$

Since $\mathcal{V} \leq 1 / 2 \mathcal{U}$, for each $j \in\{0, \ldots, n-1\}$ there exists $U_{j+1} \in \mathcal{U}$ with $V^{j+1} \cup V_{i(j+1)} \subset$ $U_{j+1}$.

By this construction, we have obtained a $(\mathcal{U}, T)$-chain $\xi$ in $\omega(x)$ given by the points $\left\{y_{0}, \ldots, y_{n}\right\} \subset \omega(x)$, times $\left\{\tau_{0}, \ldots, \tau_{n-1}\right\} \subset \mathbb{R}^{+}$with $\tau_{0}=\ldots=\tau_{n-2}=T, \tau_{n-1}=$ $r \geq T$, and sets $\left\{U_{1}, \ldots, U_{n}\right\} \subset \mathcal{U}$, and it holds that

$$
\begin{aligned}
\|\lambda-\rho(\xi)\| & \leq\|\lambda-\rho(\widetilde{\xi})\|+\|\rho(\widetilde{\xi})-\rho(\xi)\| \\
& \leq \frac{14}{2}+\frac{1}{T_{0}} \sum_{j=0}^{n-1}\left\|\tau_{j} \rho^{\tau_{j}}\left(x_{j}\right)-\tau_{j} \rho^{\tau_{j}}\left(y_{j}\right)\right\| \\
& \leq \frac{11}{2}+\frac{n}{T_{0}} \frac{\delta}{2} \delta,
\end{aligned}
$$

which finishes the proof.

Remark 6 We like to note that in the setting of the preceding theorem, the restriction of the semiflow $\Phi$ to the $\omega$-limit set $\omega(x)$ is chain transitive (cf. Patrão \& San Martin [11. Cor. 3.15]).

\section{Open Questions}

We end this paper with a list of unsolved problems and topics for further research which we want to investigate in the future.

1. We would like to know more about the topological and geometric properties of the uniform growth spectrum. For instance, for discrete-time systems we do not know if this set is connected provided that $\mathcal{M}$ is connected. Another topic for further research concerns the dimensions of the spectral sets $\Sigma_{\mathrm{Ly}}(\mathcal{M}), \Sigma_{\mathrm{UG}}(\mathcal{M})$ and $\Sigma_{\mathrm{Mo}}(\mathcal{M})$.

2. A further open question concerns the dependence of the spectral sets $\Sigma_{\mathrm{UG}}(\mathcal{M})$ and $\Sigma_{\mathrm{Mo}}(\mathcal{M})$ on flow parameters. In Colonius \& Kliemann [2, Thm. 3.11], upper semicontinuous dependence of the Morse spectrum under appropriate assumptions is proved for a family of linear flows on vector bundles. However, for the Morse spectrum in the general setting of the present paper, this is still an unsolved problem.

3. In Remark 3 we pointed out the relations between the different spectral sets. However, in the general setting, we do not know if or when equality holds in the inclusions $\Sigma_{\mathrm{Ly}}(\mathcal{M}) \subset \Sigma_{\mathrm{UG}}(\mathcal{M})$ and $\Sigma_{\mathrm{UG}}(\mathcal{M}) \subset \Sigma_{\mathrm{Mo}}(\mathcal{M})$. 


\section{Acknowledgements}

This paper essentially contains results of the second author's Ph.D. thesis, which was supervised by Fritz Colonius. We thank Fritz Colonius for many helpful discussions, and Lars Grüne for helping us solving a technical problem.

\section{References}

1 Colonius, F., Fabbri, R., and Johnson, R. (2007). Chain recurrence, growth rates and ergodic limits. Ergodic Theory Dynam. Systems 27, 1509-1524.

2 Colonius, F., Kliemann W. (1996). The Morse spectrum of linear flows on vector bundles. Trans. Amer. Math. Soc. 348, 4355-4388.

3 Colonius, F., Kliemann, W. (2000). The Dynamics of Control, Birkhäuser, Boston.

4 Conley C. (1978). Isolated Invariant Sets and the Morse Index, Regional Conference Series in Mathematics, No. 38. American Mathematical Society, Providence.

5 Dugundji, J. (1966). Topology, Series in Advanced Mathematics, Allyn and Bacon, Boston.

6 Grüne, L. (2000). A uniform exponential spectrum for linear flows on vector bundles. J. Dynam. Differential Equations 12, 435-448.

7 Johnson, J., Moser, J. (1982). The rotation number for almost periodic potentials. Commun. Math. Phys. 84, 403-438; erratum ibid. 90 (1983), 317-318.

8 San Martin, L. A. B., Seco L. (2010). Morse and lyapunov spectra and dynamics on flag bundles. Ergod. Th. \& Dynam. Sys. 30, 893-922.

9 Mezić, I., Banaszuk, A. (2004). Comparison of systems with complex behavior. Phys. D 197, 101-133.

10 Patrão, M. (2007). Morse decomposition of semiflows on topological spaces. J. Dynam. Differential Equations 19, 181-198.

11 Patrão, M., San Martin, L. A. B. (2007). Semiflows on topological spaces: chain transitivity and semigroups. J. Dynam. Differential Equations 19, 155-180.

12 Patrão M., San Martin L. A. B. (2007). Morse decomposition of semiflows on fiber bundles. Discrete Contin. Dyn. Syst. 17, 561-587.

13. San Martin L. A. B. (1988). Rotation numbers in higher dimensions. Report 199, Institut für Dynamische Systeme, Bremen.

14 Stender, T. (2007). A generalization of imaginary parts of eigenvalues for matrices: chain rotation numbers. Linear Algebra Appl. 426, 53-70.

15. Stender, T. (2009). Growth Rates for Semiflows with Application to Rotation Numbers for Control Systems (doctoral thesis), Augsbg. Schr. Math. Phys. Inform., Augsburg.

16. Wichtrey, T. (2010). Harmonic Limits of Dynamical and Control Systems (doctoral thesis), Augsb. Schr. Math. Phys. Inform., Augsburg.

17 Willard, S. (1970). General Topology, Addison-Wesley Publishing Co., Reading, Mass.London-Don Mills, Ont. 